\title{
Stability analysis of rapid granular chute flows: formation of longitudinal vortices.
}

\author{
By YOËL FORTERRE AND OLIVIER POULIQUEN \\ IUSTI, Technopôle Château-Gombert, 5 rue Enrico Fermi, 13453 Marseille Cedex 13, France.
}

(Received 2001)

In a recent article (Forterre \& Pouliquen 2001), we have reported a new instability observed in rapid granular flows down inclined planes that leads to the spontaneous formation of longitudinal vortices. From the experimental observations, we have proposed an instability mechanism based on the coupling between the flow and the granular temperature in rapid granular flows. In order to investigate the relevance of the proposed mechanism, we perform in the present paper a three-dimensional linear stability analysis of steady uniform flows down inclined planes using the kinetic theory of granular flows. We show that in a wide range of parameters, steady uniform flows are unstable under transverse perturbations. The structure of the unstable modes are in qualitative agreement with the experimental observations. This theoretical analysis shows that the kinetic theory is able to capture the formation of longitudinal vortices and validates the instability mechanism.

\section{Introduction}

Unlike classical fluids which are well-described by Navier-Stokes constitutive equations, granular flows still lack an unifying description. For slow deformations at high density, multi-body interactions and friction between grains control the dynamics of the flow. On the other hand, when the energy injected into the material is large, the particles are strongly agitated and interact mainly via instantaneous collisions. In this collisional regime, the material can be compared to a gas and a "granular temperature" can be defined in relation with the random motion of the particles (Ogawa et al 1980. Campbell 1990). This analogy between a collisional granular flow and a molecular gas has led to the development of a kinetic theory for rapid granular flows (Jenkins \& Savage 1983; Haff 1983; Lun et al 1984; Brey et al 1998: Sela \& Goldhirsch 1998) which is inspired by the kinetic theory of dense gas (Chapman \& Cowling 1970). However, the main difference with classical molecular gases is that the collisions between granular particles are inelastic. If no energy is supplied to the system, the granular temperature decays rapidly because each collision removes kinetic energy from the particles. It can be shown that a free dissipative gas can form dense clusters and eventually collapses in a finite time, as a consequence of inelasticity (McNamara \& Young 1992; Goldhirsch \& Zanetti 1993). In order to maintain the collisional regime, energy must therefore be supplied to the system, for instance by strongly shaking the boundaries (Warr et al 1995; Falcon et al 1999; Rouyer \& Menon 2000. In a rapid granular flow, another way to maintain granular temperature is to impose a shear to the mean flow. In that case, the granular temperature results from a balance between the production by the shear work and the lost due to the inelastic collisions. In return, the granular temperature influences the mean flow because the pressure and the transport coefficients (e.g. viscosity, thermal conductivity) 
depend on the temperature, as in a molecular gas. The coupling between the granular temperature and the mean flow is one of the fundamental properties of rapid granular flows. Understanding the role of this coupling is thus important in order to better define the analogies and the differences between classical fluid and granular flows.

Recently, we have reported an instability observed in rapid granular flows down rough inclined planes which seems to result from this coupling (Forterre \& Pouliquen 2001). The experimental set-up was a rough inclined plane as sketched in figure 11(a). For high inclinations and large openings of the reservoir, the free surface deforms in a very regular pattern of longitudinal streaks parallel to the flow direction (see the picture in figure 1 la). Velocity measurements of the grains have revealed that the streaks correspond to the formation of longitudinal vortices as sketched in figure 1(b). Although such structures are common in fluid mechanics (e.g. Grtler vortices, see Saric 1994 or streaks in turbulent boundary layers, see Kachanov 1994, they had not been observed before in granular flows. The main experimental observations are the following:

- The wavelength $\lambda$ of the surface deformation scales with the mean thickness $h$ of the flow $(\lambda \sim 2-3 h)$.

- The troughs correspond to downward part of the flow while the crests correspond to upward part of the flow (see figure $1 \mathrm{~b}$ ).

- The $x$-component of velocity is greater in the troughs than in the crests.

- Troughs are dense whereas crest are dilute (see figure 11b).

- The pattern drifts slowly in the transverse direction.

These observations suggest the following instability mechanism to explain the formation of the longitudinal vortices (Forterre \& Pouliquen 2001). Because of the collisions with the rough bottom, particles close to the plane are strongly agitated i.e. the granular temperature is high at the bottom. Since high temperature means low density, density may become lower at the bottom than at the free surface. The flow is then mechanically unstable under gravity yielding longitudinal vortices. The mechanism we propose is analogous to the classical Rayleigh-Bénard instability observed when a fluid is heated from below. However, in the present case the heating is not imposed by a thermostat but is created by the flow itself through the coupling between temperature and shear specific to granular media.

The above explanation for the vortices formation is based on density profile inversion. In a granular dissipative gas, the density profile actually results from a complex balance between gravity, collisions and dissipation and its prediction is not straightforward. Density profiles with higher density at the free surface than below have been observed in numerical simulations of rapid granular flows using discrete element methods (Campbell \& Brennen 1985; Azanza 1998). However, no instability was observed because the simulations were two-dimensional. In the present paper, we want to investigate the relevance of the proposed instability mechanism by using the kinetic theory of granular flows. We present a linear stability analysis of steady uniform flows down inclined planes, in the framework of the classical kinetic theory of Lun et al (1984). This theory provides a set of hydrodynamic equations coupling the density, the velocity and the granular temperature under the assumption of instantaneous binary inelastic collisions. Although steady uniform flows down inclined planes have been studied within this framework (Anderson \& Jackson 1992; Ahn et al 1992; Azanza et al 1999), no stability analysis has been performed. Stability studies of rapid granular flows with the kinetic theory have mainly focused on two-dimensional Couette flows Savage 1992. Chi-Hwa Wang et al 1997; Alam \& Nott 1998; Nott et al 1999.

We do not expect from the present study a complete and quantitative description of the longitudinal vortices instability, since the applicability of the kinetic theory is 


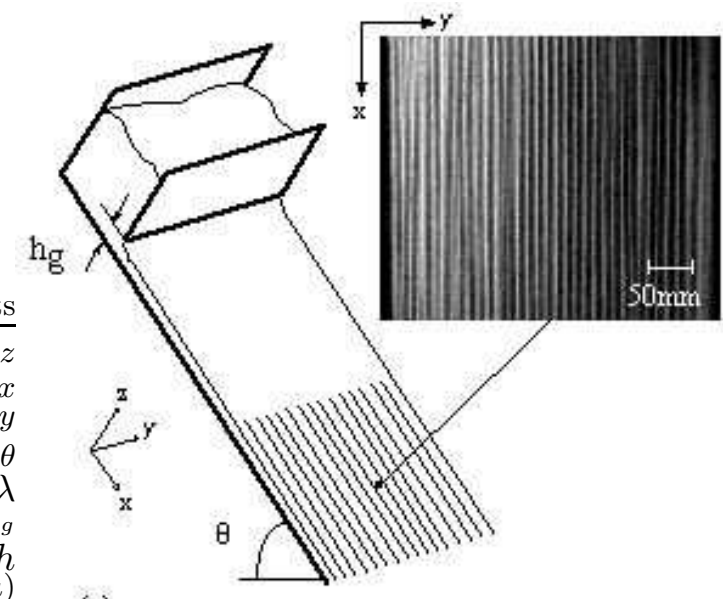

(a)

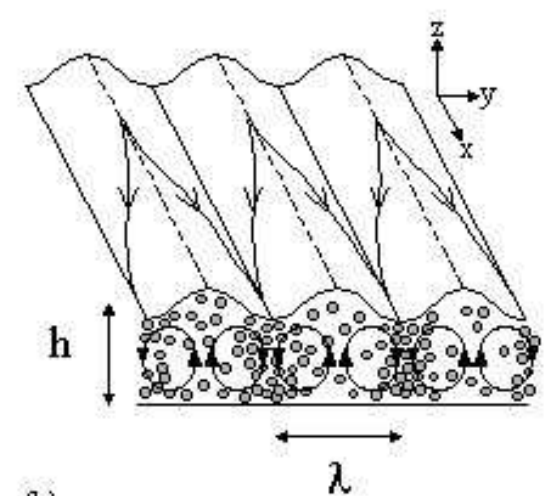

(b)

Figure 1. (a) Experimental set-up. The picture is a top view of the free surface lit from side when the instability is fully developed (the granular material is sand $0.25 \mathrm{~mm}$ in mean diameter, the angle of inclination $\theta$ of the plane is $\theta=41^{\circ}$, the opening $h_{g}$ of the reservoir is $h_{g}=13$ $\mathrm{mm}$ ). (b) Sketch of the flow deduced from the measurements showing the surface deformation, the longitudinal vortices and the density variations.

known to be limited. The lack of separation between the microscopic and macroscopic scales inherent to inelastic gases (Tan \& Goldhirsch 1999) and the existence of multibody interactions when density is high, are serious difficulties which are the subject of active researches Goldhirsch 1999). However, the kinetic theory qualitatively contains the most important character of rapid granular flow i.e. the coupling between shear flow and particle agitation due to the inelasticity of collisions. This coupling being the core of the instability mechanism we propose, this analysis should capture at least qualitatively the formation of longitudinal vortices.

The paper is structured as follow. The Section 2 gives the governing equations and the boundary conditions we will use in the paper. Basic flows i.e. steady uniform flows down inclined planes are studied in Section 3. Section 4 is devoted to the linearization of the equations around the basic state and the numerical method. The results of the stability analysis are given in Section 5 . Comparison with the experiment and discussion are presented in Section 6. Concluding remarks are given in Section 7.

\section{Kinetic theory of granular flows}

In this section, we recall the equations of the kinetic theory of granular flow we will use to investigate the formation of longitudinal vortices.

\subsection{Governing equations}

The kinetic theory of granular flows provides a set of hydrodynamic equations coupling the density $\rho$, the mean velocity $\mathbf{u}$, and the granular temperature $T$ under the assumption of instantaneous binary inelastic collisions (the granular temperature $T$ is defined by $\frac{1}{3}<\delta \mathbf{u}^{2}>$, where $\delta \mathbf{u}$ is the random velocity fluctuations). In the presence of gravity, the hydrodynamic equations are:

$$
\frac{\mathrm{d} \rho}{\mathrm{d} t}=-\rho \nabla \cdot \mathbf{u}
$$




$$
\begin{aligned}
\rho \frac{\mathrm{d} \mathbf{u}}{\mathrm{d} t} & =\rho \mathbf{g}+\nabla \cdot \boldsymbol{\Sigma}, \\
\frac{3}{2} \rho \frac{\mathrm{d} T}{\mathrm{~d} t} & =\boldsymbol{\Sigma}: \nabla \mathbf{u}-\nabla \cdot \mathbf{q}-\gamma,
\end{aligned}
$$

where $\mathrm{d} / \mathrm{d} t=\partial / \partial t+\mathbf{u} . \nabla$. The first equation (2.1) is the conservation of mass. The second equation (2.2) comes from the conservation of momentum, where $\boldsymbol{\Sigma}$ is the stress tensor and $\mathbf{g}$ is the gravity acceleration. The third equation (2.3) is the energy equation where the temporal variation of the random kinetic energy is balanced by three terms. The term $\boldsymbol{\Sigma}: \nabla \mathbf{u}$ represents the production of fluctuation energy due to the work of the stress $\boldsymbol{\Sigma}$ during shear. The term $-\nabla . \mathbf{q}$, where $\mathbf{q}$ is the flux of fluctuation energy, represents the conduction term. The term $\gamma$ is the collisional rate of energy dissipation. The distinctive feature of rapid granular flows lies in the dissipative term $\gamma$ due to the inelastic collisions between particles. This last term is responsible for the coupling between the mean flow and the granular temperature.

The kinetic theory gives the constitutive relations for $\boldsymbol{\Sigma}$, $\mathbf{q}$ and $\gamma$ as a function of the flow variables $\rho, \mathbf{u}$ and $T$. For the present purpose, we will use the closure due to Lun et al (1984). The total stress tensor $\boldsymbol{\Sigma}$, the heat flux $\mathbf{q}$ and the rate of energy dissipation $\gamma$ are written as:

$$
\begin{aligned}
\boldsymbol{\Sigma} & =-\{P(\nu, T)-\xi(\nu, T) \nabla \cdot \mathbf{u}\} \mathbf{I}+2 \eta(\nu, T) \mathbf{S} \\
\mathbf{q} & =-K(\nu, T) \nabla T \\
\gamma & =\frac{\rho_{p}}{d}\left(1-e^{2}\right) f_{5}(\nu) T^{\frac{3}{2}}
\end{aligned}
$$

Here $\mathbf{I}$ is the identity matrix, $\mathbf{S}=\frac{1}{2}\left(u_{i j}+u_{j i}\right)-\frac{1}{3} u_{k k} \delta_{i j}$ is the deviatoric part of the rate of deformation, $\rho_{p}$ is the particle density, $\nu=\rho / \rho_{p}$ is the solid fraction and $d$ is the particle diameter. We have omitted in (2.5) the contribution of the gradient of compacity to the heat flux. This term increases considerably the algebra while it has little effect on the qualitative nature of the results. Note that the collisional rate of energy dissipation $\gamma$ is proportional to $\left(1-e^{2}\right)$, where $e$ is the coefficient of inelasticity of the particles $(0<e \leq 1)$. As in classical dense gases, the pressure $P(\nu, T)$, the viscosities $(\eta(\nu, T)$, $\xi(\nu, T))$ and the thermal conductivity $K(\nu, T)$ depend on the solid fraction $\nu$ and the temperature $T$ :

$$
\left\{\begin{array}{l}
P(\nu, T)=\rho_{p} f_{1}(\nu) T, \\
\eta(\nu, T)=\rho_{p} d f_{2}(\nu) T^{\frac{1}{2}}, \\
\xi(\nu, T)=\rho_{p} d f_{0}(\nu) T^{\frac{1}{2}}, \\
K(\nu, T)=\rho_{p} d f_{3}(\nu) T^{\frac{1}{2}},
\end{array}\right.
$$

where the dimensionless functions $f_{0}(\nu), f_{1}(\nu), f_{2}(\nu), f_{3}(\nu)$ and $f_{5}(\nu)$ are given in table 1 (Lun et al 1984). These functions involve the radial distribution function $g_{0}(\nu)$. In the following, we shall used the one suggested by Lun and Savage (1986):

$$
g_{0}(\nu)=\frac{1}{\left(1-\frac{\nu}{\nu_{m}}\right)^{2.5 \nu_{m}}},
$$

where $\nu_{m}$ is the maximum solid fraction $\left(\nu_{m}=0.6\right.$ in the following). This radial distribution function is suitable for free surface flows since the resulting equations have no singularity at $\nu=0$. We have checked that this choice does not change qualitatively the results by using other kind of radial distribution functions, such as the Carnahan-Stirling radial distribution (Jenkins \& Savage 1983). Finally, all the equations that follow will 


$$
\begin{aligned}
& f_{0}(\nu)=\frac{8 \sqrt{\pi}}{3} \eta \nu^{2} g_{0}(\nu), \\
& f_{1}(\nu)=\nu+4 \eta \nu^{2} g_{0}(\nu), \\
& f_{2}(\nu)=\frac{5 \sqrt{\pi}}{96}\left[\frac{1}{\eta(2-\eta)} \frac{1}{g_{0}(\nu)}+\frac{8}{5} \frac{3 \eta-1}{2-\eta} \nu+\frac{64}{25} \eta\left(\frac{3 \eta-2}{2-\eta}+\frac{12}{\pi}\right) \nu^{2} g_{0}(\nu)\right], \\
& f_{3}(\nu)=\frac{25 \sqrt{\pi}}{16 \eta(41-33 \eta)}\left[\frac{1}{g_{0}(\nu)}+\frac{12}{5} \eta(1+\eta(4 \eta-3)) \nu+\frac{16}{25} \eta^{2}\left(9 \eta(4 \eta-3)+\frac{4}{\pi}(41-33 \eta)\right) \nu^{2} g_{0}(\nu)\right], \\
& f_{5}(\nu)=\frac{12}{\sqrt{\pi}} \nu^{2} g_{0}(\nu), \\
& f_{6}(\nu)=\frac{\pi \sqrt{3}}{6 \nu_{m}} \nu g_{0}(\nu), \\
& f_{7}(\nu)=\frac{3 \sqrt{3} \pi}{12 \nu_{m}} \nu g_{0}(\nu),
\end{aligned}
$$

TABLE 1. Dimensionless constitutive functions. $\eta=\frac{1}{2}(1+e)$

be written in term of non-dimensional variables defined by:

$$
(\tilde{x}, \tilde{y}, \tilde{z})=\frac{1}{d}(x, y, z), \tilde{t}=\sqrt{\frac{d}{g}} t, \tilde{\nu}=\frac{\rho}{\rho_{p}}, \tilde{\mathbf{u}}=\frac{1}{\sqrt{g d}} \mathbf{u}, \tilde{T}=\frac{1}{g d} T .
$$

For sake of simplicity in the notation, the tildes will be omitted and the solid fraction $\nu$ will be called density in the following .

\subsection{Boundary conditions}

In order to solve the problem of granular flows down rough inclined planes, we have to specify boundary conditions for $\nu, \mathbf{u}$ and $T$ both at the free surface of the flow and at the plane. Unlike classical fluid, the velocity in rapid granular flows does not vanish at a fixed solid boundary. Therefore, the rough plane may act as a source (resp. a sink) of fluctuating energy whether the shear work of the slip velocity is larger (resp. smaller) than the inelastic loss due to collisions with the plane. Boundary conditions for rapid granular flows at a rough surface have been the subject of extensive researches (Hui et al 1984; Jenkins \& Richman 1986; Johnson et al 1990; Goldhirsch 1999). Here we will use the heuristic approach of Johnson et al (1990) relating the tangential stresses t. $\boldsymbol{\Sigma} . \mathbf{n}$ and the heat flux $\mathbf{q} \cdot \mathbf{n}$ at the plane to density, slip velocity $\mathbf{u}_{\mathbf{s}}$ and temperature by:

$$
\begin{aligned}
\text { t. } \boldsymbol{\Sigma} \cdot \mathbf{n} & =\eta^{*}(\nu, T)\left\|\mathbf{u}_{\mathbf{s}}\right\|, \quad \text { at the plane, } \\
\mathbf{q} \cdot \mathbf{n} & =(\mathbf{u} \cdot \boldsymbol{\Sigma}) \cdot \mathbf{n}-\gamma^{*}(\nu, T), \quad \text { at the plane. }
\end{aligned}
$$

The vector $\mathbf{t}$ is parallel to the plane and defined as $\mathbf{t}=\mathbf{u}_{\mathbf{s}} /\left\|\mathbf{u}_{\mathbf{s}}\right\|$. The vector $\mathbf{n}$ is normal to the plane. Finally the function $\eta^{*}(\nu, T)$ and $\gamma^{*}(\nu, T)$ are given by:

$$
\left\{\begin{array}{l}
\eta^{*}(\nu, T)=\phi f_{6}(\nu) T^{\frac{1}{2}} \\
\gamma^{*}(\nu, T)=\left(1-e_{w}{ }^{2}\right) f_{7}(\nu) T^{\frac{3}{2}}
\end{array}\right.
$$

where the dimensionless functions $f_{6}(\nu)$ and $f_{7}(\nu)$ are given in table 1. The relation (2.10) is a transfer of momentum balance at the plane. Equation 2.11) expresses that 
heat can be produced at the plane if the shear work is stronger than the loss of energy due to collisions with the plane. The boundary conditions (2.10) and (2.11) depend on two dimensionless parameters, $\phi$ and $e_{w}$, which are related to the wall properties. The parameter $e_{w}$ is the particle-wall coefficient of restitution. In the following, $e_{w}$ will be taken equal to the particle-particle coefficient of restitution $e$. The parameter $\phi$ is related to the rate of momentum transfer to the flow by the collision with the plane. Its value can be connected to the wall geometry in the case of $2 \mathrm{D}$-flows of disk (Jenkins \& Richman 1986). For a rough plane made of close-packed disks, one obtains a value $\phi \sim 0.1$. For 3D-flows, one can expect lower values of $\phi$ since collisions do not always occur in the shear plane. We will use in the following the value $\phi=0.05$ and will discuss its influence later.

At the free surface, the stresses and the energy flux must vanish. However, the location of the free surface is not known a priori and its definition is somewhat artificial for very dilute flows. Rather than define the location of the free surface, we impose stresses and energy flux to vanish when the distance from the plane goes to infinity. It should be notice that the boundary conditions used here are somewhat different than those used in previous studies (Johnson et al 1990; Anderson \& Jackson 1992; Ahn et al 1992; Azanza et al 1999). In the work of Anderson \& Jackson (1992), the thickness $h$ of the granular layer is taken as a control parameter. Numerical difficulties then arise when trying to match the stress-free condition at the free surface (Johnson et al 1990). Ahn et al (1992) do not define the free surface but impose stresses to vanish at infinity. However, they arbitrarily fixe the density, velocity and granular temperature at the plane. The same procedure is used by Azanza et al (1999). Here, we adopt a mixed point of view since at the plane the boundary conditions of Johnson et al (1990) are satisfied whereas at infinity the procedure of Ahn et al (1992) is chosen.

\section{Steady uniform flows}

The first step in order to perform a linear stability analysis is to determine the basic flow i.e. two-dimensional steady uniform flows. Steady uniform flows down inclined planes have already been studied in the framework of the kinetic theory (Anderson \& Jackson 1992; Ahn et al 1992; Azanza et al 1999). It is not in the scope of the present study to make an extensive investigation of steady uniform flows. Rather, we shall focus on the shape of the density profile, which plays an important role in the instability mechanism we propose.

\subsection{Equations for steady uniform flows}

We apply equations (2.1)-(2.3) with boundary conditions (2.10) and 2.11) to twodimensional steady uniform flows down inclined planes. We thus look for solution for density, velocity and temperature in the following form:

$$
\left\{\begin{array}{l}
\nu(x, y, z, t)=\nu_{0}(z) \\
\mathbf{u}(x, y, z, t)=u_{0}(z) \mathbf{x} \\
T(x, y, z, t)=T_{0}(z)
\end{array}\right.
$$

In such a flow the derivatives parallel to the plane are zero and the mass-conservation equation (2.1) is satisfied. The momentum-conservation equation (2.2) in the flow direc- 
Stability analysis of rapid granular chute flows: formation of longitudinal vortices. 7 tion ( $x$-direction) and in the direction normal to the plane (z-direction) becomes:

$$
\begin{aligned}
& \frac{\mathrm{d} \Sigma_{x z 0}}{\mathrm{~d} z}=-\nu_{0} \sin \theta, \\
& \frac{\mathrm{d} \Sigma_{z z 0}}{\mathrm{~d} z}=\nu_{0} \cos \theta,
\end{aligned}
$$

where $\theta$ is the angle of inclination of the plane, $\Sigma_{x z 0}=f_{2}\left(\nu_{0}\right) T_{0}{ }^{1 / 2} \mathrm{~d} u_{0} / \mathrm{d} z$ and $\Sigma_{z z 0}=$ $-f_{1}\left(\nu_{0}\right) T_{0}$. The energy-conservation (2.3) simplifies to:

$$
0=\Sigma_{x z 0} \frac{\mathrm{d} u_{0}}{\mathrm{~d} z}-\frac{\mathrm{d} q_{z_{0}}}{\mathrm{~d} z}-\gamma_{0},
$$

where $q_{z 0}=-f_{3}\left(\nu_{0}\right) T_{0}{ }^{1 / 2} \mathrm{~d} T_{0} / \mathrm{d} z$ and $\gamma_{0}=\left(1-e^{2}\right) f_{5}\left(\nu_{0}\right) T_{0}{ }^{3 / 2}$.

From (3.2) and (3.3) together with boundary conditions $\Sigma_{x z 0}=\Sigma_{z z 0}=0$ at infinity, one obtains $\Sigma_{x z 0}=-\tan \theta \Sigma_{z z 0}$. Therefore, equations (3.2), (3.3) and (3.4) can be written in the following form:

$$
\begin{aligned}
\frac{\mathrm{d} \nu_{0}}{\mathrm{~d} z} & =-\frac{1}{f_{1}{ }^{(}\left(\nu_{0}\right) T_{0}}\left(\nu_{0} \cos \theta+f_{1}\left(\nu_{0}\right) \frac{\mathrm{d} T_{0}}{\mathrm{~d} z}\right) \\
\frac{\mathrm{d} u_{0}}{\mathrm{~d} z} & =\frac{f_{1}\left(\nu_{0}\right)}{f_{2}\left(\nu_{0}\right)} \tan \theta T_{0}{ }^{\frac{1}{2}}, \\
\frac{\mathrm{d}^{2} T_{0}}{\mathrm{~d} z^{2}} & =\left(\frac{\left(1-e^{2}\right) f_{5}\left(\nu_{0}\right) f_{2}\left(\nu_{0}\right)-f_{1}{ }^{2}\left(\nu_{0}\right) \tan \theta}{f_{2}\left(\nu_{0}\right) f_{3}\left(\nu_{0}\right)}\right) T_{0}+\frac{\nu_{0} f_{3}{ }^{\prime}\left(\nu_{0}\right)}{f_{3}\left(\nu_{0}\right) f_{1}{ }^{\prime}\left(\nu_{0}\right) T_{0}} \cos \theta \frac{\mathrm{d} T_{0}}{\mathrm{~d} z} \\
& +\frac{1}{T_{0}}\left(\frac{f_{1}\left(\nu_{0}\right) f_{3}{ }^{\prime}\left(\nu_{0}\right)}{f_{3}\left(\nu_{0}\right) f_{1}{ }^{\prime}\left(\nu_{0}\right)}-\frac{1}{2}\right)\left(\frac{\mathrm{d} T_{0}}{\mathrm{~d} z}\right)^{2},
\end{aligned}
$$

where the dashes in $f_{1}{ }^{\prime}$ and $f_{3}{ }^{\prime}$ denote differentiation with respect to $\nu_{0}$.

This system of three nonlinear ordinary differential equations requires boundary conditions both at the plane and at infinity. At infinity, the stresses and the flux of energy must vanish. It can be shown Ahn et al 1992 that these conditions are satisfied if and only if the temperature gradient vanishes at infinity:

$$
\frac{\mathrm{d} T_{0}}{\mathrm{~d} z} \rightarrow 0 \quad \text { when } z \rightarrow \infty .
$$

At the plane, the relations (2.10) and (2.11) become:

$$
\begin{aligned}
f_{2}\left(\nu_{0}\right) T_{0} \frac{1}{2} \frac{\mathrm{d} u_{0}}{\mathrm{~d} z} & =\phi f_{6}\left(\nu_{0}\right) T_{0}^{\frac{1}{2}} u_{0} \quad \text { at } z=0, \\
-f_{3}\left(\nu_{0}\right) T_{0} \frac{1}{2} \frac{\mathrm{d} T_{0}}{\mathrm{~d} z} & =f_{2}\left(\nu_{0}\right) T_{0}^{\frac{1}{2}} \frac{\mathrm{d} u_{0}}{\mathrm{~d} z} u_{0}-\left(1-e^{2}\right) f_{7}\left(\nu_{0}\right) T_{0} \frac{3}{2} \quad \text { at } z=0 .
\end{aligned}
$$

These relations can be combined with equation (3.6) to express the temperature and the gradient of temperature at the plane as a function of density and velocity at the plane:

$$
\begin{aligned}
T_{0} & =\frac{\phi^{2} f_{6}{ }^{2}\left(\nu_{0}\right)}{f_{1}^{2}\left(\nu_{0}\right) \tan ^{2} \theta} u_{0}^{2} \quad \text { at } z=0, \\
\frac{\mathrm{d} T_{0}}{\mathrm{~d} z} & =\frac{\phi f_{6}\left(\nu_{0}\right)}{f_{3}\left(\nu_{0}\right)}\left(-1+\frac{\phi f_{6}\left(\nu_{0}\right)\left(1-e^{2}\right) f_{7}\left(\nu_{0}\right)}{f_{1}{ }^{2}\left(\nu_{0}\right) \tan ^{2} \theta}\right) u_{0}^{2} \quad \text { at } z=0 .
\end{aligned}
$$

In order to integrate numerically the above boundary value problem, a "shooting method" has been chosen. The angle $\theta$ and the density at the wall $\nu_{0}(0)$ are the two control parameters whereas the slip velocity $u_{s}=u_{0}(0)$ is taken as the shooting parameter. Then, 


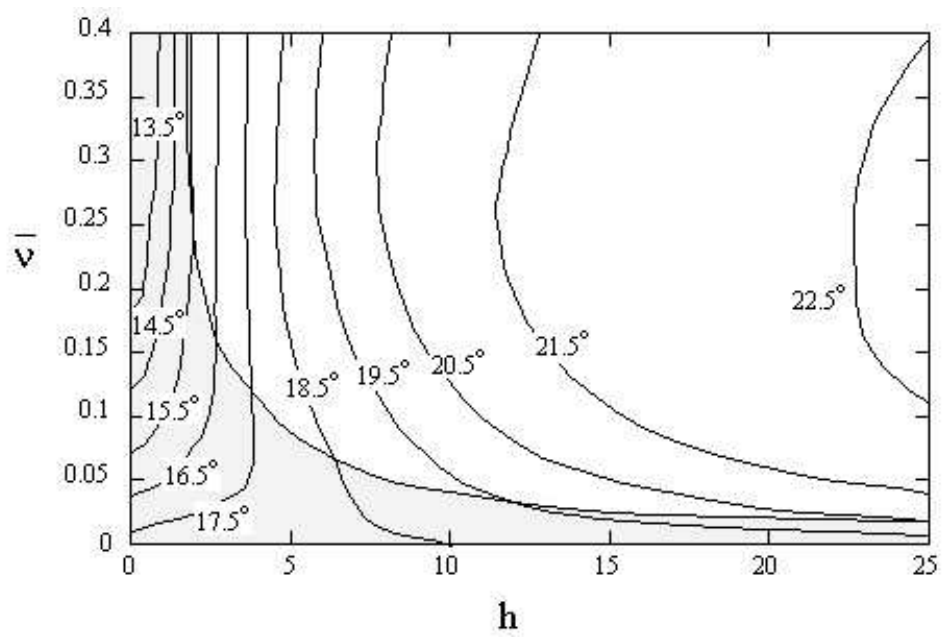

Figure 2. Phase diagram for steady uniform flows in the $(h, \bar{\nu})$ plane. The thin solid lines give the contours of constant angle $\theta$. The grey zone is the domain of non-inverted density profiles. $(e=0.6, \phi=0.05)$.

the temperature and the gradient of temperature at the plane are given by the equations (3.11) and (3.12). The differential equations (3.5)-(3.7) can therefore be integrated from $z=0$ to $z=\infty$. This procedure is repeated for different values of the shooting parameter $u_{s}=u_{0}(0)$ until the boundary condition at infinity $\mathrm{d} T_{0} / \mathrm{d} z=0$ is satisfied. In practice, a fourth-order Runge-Kutta method is employed and the equations are integrated up to a maximum value $z=z_{\max }$. The boundary condition at infinity is considered to be satisfied when the gradient of temperature is smaller than $10^{-5}$ at $z=z_{\max }$.

\subsection{Results}

By applying the above procedure to different values of the angle $\theta$ and the density $\nu_{0}(0)$, we were able to explore the whole set of steady uniform flows. However, in order to help the physical interpretation of the results, we present the results in the $(h, \bar{\nu})$ plane where $h$ is the thickness of the flow and $\bar{\nu}$ the mean density. The thickness $h$ is defined $a$ posteriori by the value $z=h$ for which the density is $1 \%$ of the maximum density inside the flow. The mean density $\bar{\nu}$ is simply given by $\bar{\nu}=(1 / h) \int_{0}^{h} \nu_{0}(z) d z$. The relation between $\left(\theta, \nu_{0}(0)\right)$ and $(h, \bar{\nu})$ is univocal i.e. a single solution exists for a given set $(h, \bar{\nu})$ (which is not the case if one chooses the flow rate as a control parameter, see Johnson et al 1990 and Anderson \& Jackson 1992). The relation between $\left(\theta, \nu_{0}(0)\right)$ and $(h, \bar{\nu})$ is shown in figure 2 for $e=0.6$ and $\phi=0.05$. In this figure, the thin solid lines are obtained for constant inclination $\theta$ by varying $\nu_{0}(0)$. We observe that the angle $\theta$ has a strong influence on the thickness of the flow $h$, the thickness of the flow rapidly increases when increasing the angle. For angles $\theta>23^{\circ}$, the thickness diverges whereas for $\theta<14^{\circ}$, the thickness becomes less than one grain diameter. Therefore, for a given set of parameters $(e, \phi)$ there exists a finite range of angles where steady uniform flows are obtained. These results are consistent with analytical solutions for steady uniform flows found in the high-density limit Anderson \& Jackson 1992).

Typical solutions $\left(\nu_{0}(z), u_{0}(z), T_{0}(z)\right)$ for steady uniform flows are given in figure 3 . They are obtained for $\theta=20.5^{\circ}$ by increasing the mean density $\bar{\nu}$ i.e. along the thin solid line $\theta=20.5^{\circ}$ in figure 3 . As the mean density is increased, the shear close to the plane increases (see figure $3 \mathrm{~b}$ ). The difference of temperature between the plane and 
Stability analysis of rapid granular chute flows: formation of longitudinal vortices. 9
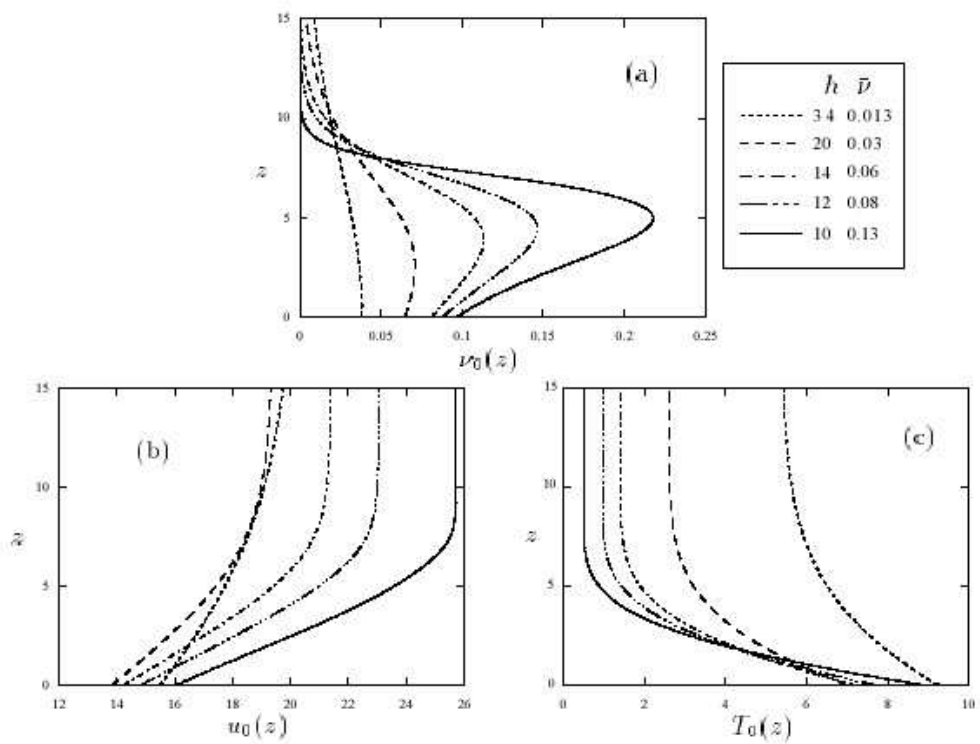

Figure 3. (a) Density profiles $\nu_{0}(z)$, (b) velocity profiles $u_{0}(z)$ and (c) temperature profiles $T_{0}(z)$ for steady uniform flows at a fixed angle $\left(\theta=20.5^{\circ}\right)$ when the mean density is increased. The inset gives the value of $(h, \bar{\nu})$ for the different profiles $(e=0.6, \phi=0.05)$.

the free surface also increases with the mean density (see figure 3ic). Note that with our boundary conditions, the temperature is always higher at the plane than at the free surface. Finally, the density profile $\nu_{0}(z)$ is strongly modified when varying the mean density (see figure 3a). For dilute flows, the thickness $h$ is large and the density profile is non-inverted i.e. the density decreases with $z$. As the mean density increases, the flow becomes thinner and the free surface is better defined. At a given mean density, the density profile becomes inverted i.e. the maximum of density is no longer at the plane but in the bulk. In the $(h, \bar{\nu})$ diagram of figure 2, the limit between non-inverted density profiles and inverted density profiles is given by the solid line. Above this line, the maximum of density is in the bulk.

The shape of density profiles can be understood qualitatively by inspecting the equations (3.5)-(3.7). $\mathrm{Eq}(3.5)$ shows that the sign of the density gradient results from a competition between gravity, which tends to increase density close to the plane, and the negative temperature gradient, which tends to decrease density close to the plane. The temperature gradient results from the shearing at the base, which heats the material from below and the inelastic dissipation in the bulk, which cools the material. Therefore, inverted density profiles are observed when heating due to the shear at the base and the cooling due to dissipation in the bulk create a temperature gradient strong enough to overcome the gravity.

\subsection{Influence of the parameters}

The results presented so far have been obtained using $e=0.6$ and $\phi=0.05$. Here we discuss briefly the qualitative influence of the inelastic coefficient $e$ and the boundary parameter $\phi$ on the solutions presented above. First, inverted density profiles are observed only for small values of $\phi$. For $\phi>0.3$, no inverted density profile can be obtained, neither by changing the control parameters $(h, \bar{\nu})$ nor by modifying the coefficient of inelasticity $e$. For high values of $\phi$, the plane is indeed no longer a source of fluctuating energy (see equation 3.12). The density gradient close to the plane is therefore negative 
and no inverted density profile can be obtained. For values of $\phi$ less than 0.3 , the results are qualitatively the same as for $\phi=0.05$. Varying $\phi$ changes the range of inclinations where steady uniform flows are observed. For example, the maximum angle is increased from $\theta \sim 23^{\circ}$ to $\theta \sim 32^{\circ}$ when increasing $\phi$ from 0.05 to $0.15(e=0.6)$. No qualitative change is also observed when changing the inelasticity $e$. Increasing the value of $e$ decreases the angles and shrinks the domain of steady uniform flows. With $e=0.8$ $(\phi=0.05)$, the range of angles is $11^{\circ}<\theta<18^{\circ}$.

Steady uniform flows with inverted density profiles can therefore be obtained in the framework of the kinetic theory of granular flows. The question we address in this study is the stability of these inverted density profiles under transverse perturbations. Obviously, gravity is a destabilizing effect since the heavy fluid is above the light one. However, gravity has to overcome the stabilizing effects due to viscosity and thermal conductivity. In a rapid granular flow, viscosity, thermal conductivity and density profiles are coupled to the flow and the prediction of the stability properties is not straightforward.

\section{Three-dimensional linear stability analysis}

We investigate the stability of steady uniform flows $\left(\nu_{0}, u_{0} T_{0}\right)$ found in the previous section using the classical normal mode analysis (Drazin \& Reid 1981). The basic flow is perturbed by infinitesimal disturbances, and their time evolution are studied by linearizing the governing equations about the basic state. The perturbations are then decomposed into different Fourier modes and because of the linearity of the governing equations, the stability of each mode can be analyzed separately.

\subsection{Normal mode analysis}

As point out by Alam \& Nott (1998), the Squire theorem does not hold for rapid granular flows since density and temperature are not constant across the layer. Therefore, the first instability is not necessary two-dimensional. In this study, we are interested in the formation of longitudinal structures and we restrict our analysis to flows which are invariant along the $x$-direction. The flow $(\nu, u, v, w, T)$ is perturbed around the basic flow $\left(\nu_{0}, u_{0}, T_{0}\right)$ and written as:

$$
\nu=\nu_{0}(z)+\nu_{1}(y, z, t), \quad\left\{\begin{array}{l}
u=u_{0}(z)+u_{1}(y, z, t) \\
v=v_{1}(y, z, t) \\
w=w_{1}(y, z, t)
\end{array}, T=T_{0}(z)+T_{1}(y, z, t),\right.
$$

where $\nu_{1},\left(u_{1}, v_{1}, w_{1}\right)$ and $T_{1}$ are respectively the density, velocity and temperature disturbances. By substituting (4.1) into the governing equations (2.1)-(2.3) and then linearizing about the basic state, we obtain a set of linear equations for $\left(\nu_{1}, u_{1}, v_{1}, w_{1}, T_{1}\right)$ (see Appendix A.1). Then, we seek normal mode solutions for density, velocity and temperature perturbations:

$$
\left(\nu_{1}, u_{1}, v_{1}, w_{1}, T_{1}\right)=\Re \mathrm{e}\left[(\hat{\nu}(z), \hat{u}(z), \hat{v}(z), \hat{w}(z), \hat{T}(z)) \mathrm{e}^{\sigma t+i k y}\right] .
$$

We have restricted the stability analysis to temporal stability i.e. the growth rate $\sigma$ is complex whereas the transverse wavenumber $k$ is assumed to be real. The basic flow $\left(\nu_{0}, u_{0}, T_{0}\right)$ is unstable under the transverse perturbation of wavenumber $k$ if the real part of the growth rate, $\Re e(\sigma)$, is positive. After some algebra, it can be shown that the perturbed variables satisfy a system of ordinary differential equations:

$$
L_{0}(z) \frac{\mathrm{d}^{2}}{\mathrm{~d} z^{2}} \hat{X}(z)+M_{0}(z) \frac{\mathrm{d}}{\mathrm{d} z} \hat{X}(z)+N_{0}(z) \hat{X}(z)=0,
$$


Stability analysis of rapid granular chute flows: formation of longitudinal vortices. 11

where $\hat{X}(z)$ is the five-elements vector defined by $\hat{X}(z)=(\hat{\nu}(z), \hat{u}(z), \hat{v}(z), \hat{w}(z), \hat{T}(z))$, and $L_{0}(z), M_{0}(z), N_{0}(z)$ are $5 \times 5$ matrices which are given in Appendix A.2. Note that these matrices depend on the basic flow $\left(\nu_{0}, u_{0}, T_{0}\right)$, on the wavenumber $k$ and on the growth rate $\sigma$. The boundary conditions at the plane can be written in the same manner:

$$
Q_{0}(z) \frac{\mathrm{d}}{\mathrm{d} z} \hat{X}(z)+R_{0}(z) \hat{X}(z)=0 \quad \text { at } z=0,
$$

where $Q_{0}(z)$ and $R_{0}(z)$ are matrices which are also given in Appendix A.2. At infinity, the disturbances $\hat{X}(z)$ have to vanish. It can be shown by doing an asymptotic expansion of equations (4.3) that the disturbances $(\hat{u}, \hat{v}, \hat{w}, \hat{T})$ decrease as $\exp (-k z)$ when $z$ is much larger than the characteristic thickness of the basic flow (see Appendix B). Matching the boundary conditions at infinity thus leads to numerical difficulties when $k$ becomes small since the computational domain varies as $1 / k$. Instead of writing the boundary conditions at infinity, the equations are integrated up to a finite value $z=z_{\max }$ and the disturbances $(\hat{u}, \hat{v}, \hat{w}, \hat{T})$ are assumed to satisfy

$$
\frac{\mathrm{d}}{\mathrm{d} z}(\hat{u}(z), \hat{v}(z), \hat{w}(z), \hat{T}(z))=-k(\hat{u}(z), \hat{v}(z), \hat{w}(z), \hat{T}(z)), \quad \text { at } z=z_{\max } .
$$

The system of five ordinary differential equations (4.3) together with the eight boundary conditions (4.4) and (4.5) constitute an eigenvalue problem. For a given basic flow $\left(\nu_{0}\right.$, $\left.u_{0}, T_{0}\right)$ and wavenumber $k$, a non-zero solution $(\hat{\nu}, \hat{u}, \hat{v}, \hat{w}, \hat{T})$ exists only for specific values of the growth rate $\sigma$.

\subsection{Numerical method}

We have solved the above eigenvalue problem thanks to a Chebychev spectral collocation method (Gottlieb et al 1984). Chebychev collocation approach has shown to be welladapted to the stability of boundary-layer flows, since Chebychev polynomials resolve the boundary regions extremely well (Malik 1990). It is then suitable for our flow which is localized close to the plane. Moreover, the use of collocation makes the derivatives easy to compute and simplifies the treatment of boundary conditions.

The principle of the Chebychev spectral collocation method is to discretize the ordinary differential equations (4.3) by interpolating the perturbed functions $(\hat{\nu}(z), \hat{u}(z), \hat{v}(z)$, $\hat{w}(z), \hat{T}(z))$ on $N+1$ collocation points given by

$$
\varsigma_{j}=\cos \frac{\pi j}{N} ; \quad(i=0, \ldots, N) .
$$

In order to relate the Chebychev space $(\varsigma \in[-1,1])$ to the physical domain $\left(z \in\left[0, z_{\max }\right]\right)$, we use a two-parameters algebric transformation Malik 1990)

$$
z=a \frac{1+\varsigma}{b-\varsigma},
$$

where $b=1+2 a / z_{\max }$ and $a=z_{h} z_{\max } /\left(z_{\max }-2 z_{h}\right)$. The location $z_{h}$ corresponds to $\varsigma=0$, i.e. half of the grid points are located in the region $0 \leq z \leq z_{h}$. This mapping allows to cluster grid points near the plane. Using the expression for the derivatives at the collocation points (Gottlieb et al 1984), the system of ordinary differential equations (4.3) together with the boundary conditions (4.4) and (4.5) reduce to a linear algebraic eigenvalue problem that can be written in the following form:

$$
\mathcal{A} \hat{\mathcal{X}}=\sigma \mathcal{B} \hat{\mathcal{X}} .
$$


Here, $\hat{\mathcal{X}}$ is the vector containing the $5(N+1)$ elements of the interpolation of $(\hat{\nu}(z), \hat{u}(z)$, $\hat{v}(z), \hat{w}(z), \hat{T}(z))$ on the $(N+1)$ collocation points:

$$
\left(\hat{\nu}_{0}, \ldots, \hat{\nu}_{N} ; \hat{u}_{0}, \ldots, \hat{u}_{N} ; \hat{v}_{0}, \ldots, \hat{v}_{N} ; \hat{w}_{0}, \ldots, \hat{w}_{N} ; \hat{T}_{0}, \ldots, \hat{T}_{N}\right)
$$

and $\mathcal{A}$ and $\mathcal{B}$ are $5(N+1) \times 5(N+1)$ matrices computed from the matrices $L_{0}, M_{0}$ and $L_{0} . \mathcal{A}$ and $\mathcal{B}$ depend on the basic flow and on the wavenumber $k$. Since the boundary conditions (4.5) do not contain the eigenvalue $\sigma$, the matrix $\mathcal{B}$ is singular. It must be noticed that the above discretization requires ten boundary conditions while the physical boundary conditions (4.4) and (4.5) give only eight relations for the perturbed fields (four at the plane and four at infinity). The same problem arises in the stability analysis of compressible hydrodynamic flows (Malik 1990). Here we have chosen the vertical momentum balance at the plane and the conservation of mass at infinity as the two extra boundary conditions.

The generalized eigenvalue problem (4.8) is solved by using the scientific software MatLab. The advantage of spectral methods is that the whole spectrum of eigenvalues may be obtained. However, many eigenvalues are spurious eigenvalues due to the discretization (Mayer \& Powel 1992). The location of these spurious modes are very sensitive to the number $N$ of collocation points while the physical modes are insensitive. This allows us to select the few physical modes among the large spectrum of the discretized problem. With a typical number of collocation points $N=50$, the absolute error in the physical eigenvalues is less than $10^{-7}$.

\section{Results}

\subsection{Diagram of stability}

Figure 1 gives the diagram of stability in the plane $(h, \bar{\nu})$ for $e=0.6$ and $\phi=0.05$. The region of non-inverted density profiles is presented on the same diagram. Since we are essentially interested in thin granular layer flows, we have limited our stability analysis to $h$; 30 particle diameters. The study is also limited to mean density less than 0.4. For higher mean density, the density profile is very sharp and the number of collocation points required for accurate computation increases dramatically.

The stability diagram can be divided in three regions: a stable region (hatched region), an unstable region with stationary modes, an unstable region with propagating modes. The stable zone is delimited by the marginal curve where the real part of $\sigma$ vanishes. One observes that for $h$ less than 7 particle diameters, the flow is always stable whatever the value of the mean density. The unstable region is composed of two regions: one where the most amplified mode is stationary i.e. $\Im \mathrm{m}(\sigma)=0$, and one where the most amplified mode is propagating i.e. with a non zero phase velocity $(\Im \mathrm{m}(\sigma) \neq 0)$. Propagating modes are obtained at low density while stationary instability takes place at high density.

The transition between the stationary instability and propagating modes appears clearly in figure 5. We have plotted in figure 5 the growth rate $\Re \mathrm{e}(\sigma)$ and $\Im \mathrm{m}(\sigma)$ as a function of the wavenumber $k$, for the most dangerous mode at different locations in the stability diagram (A, B and C in figure 1 ). We keep the thickness constant ( $h=12.8$ ) and increase the mean density from $\bar{\nu}=0.08$ to $\bar{\nu}=0.16$. One sees that the stationary mode in $\mathrm{C}$ appears from the collapse of the two conjugated propagating modes. In figure 5 we also observe that at the threshold of the instability, the first unstable mode occurs at a finite wavenumber $k_{c}$. We have systematically studied the critical wavelength $\lambda_{c}=2 \pi / k_{c}$ along the marginal curve. When the instability is stationary, the wavelength $\lambda_{c}$ is nearly constant and equal twice the thickness of the flow. By contrast, the wave- 
Stability analysis of rapid granular chute flows: formation of longitudinal vortices. 13

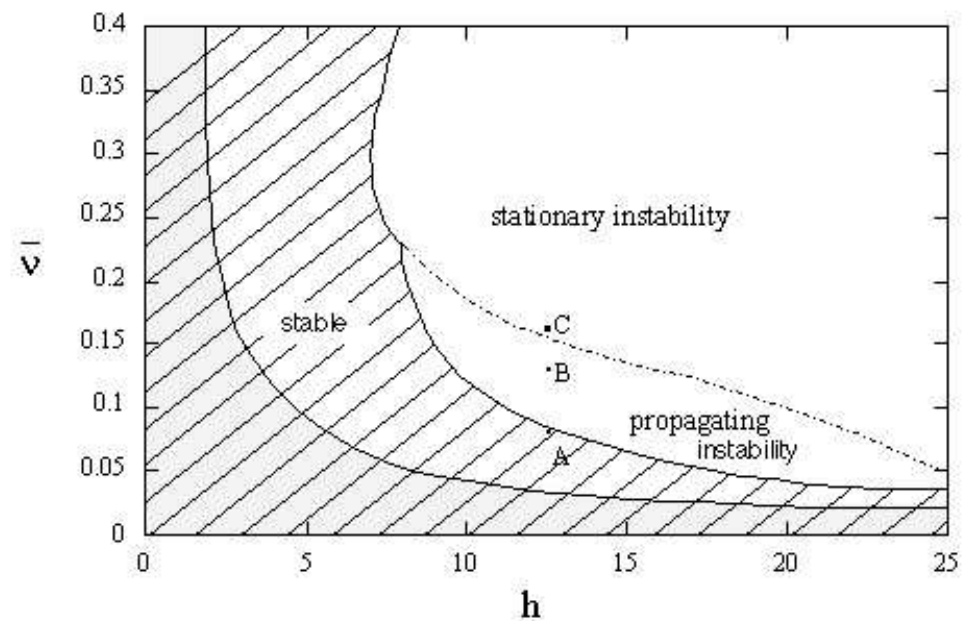

Figure 4. Stability diagram for $e=0.6$ and $\phi=0.05$. The flow is stable inside the hatched region and unstable outside. The bold solid line is the marginal curve. The dotted line separates a region where the most amplified mode is propagating from a region where it is stationary. The grey zone is the domain of non-inverted density profiles.
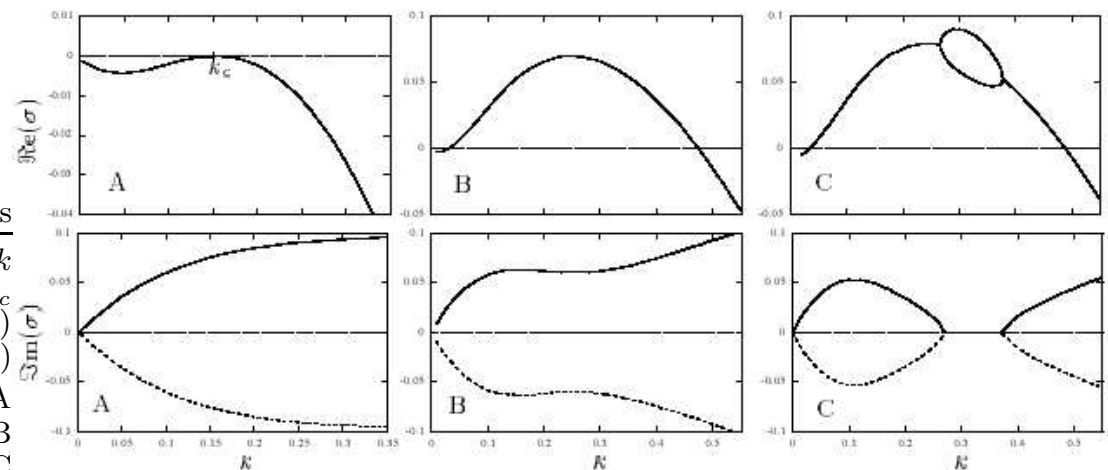

Figure 5. Growth rate $\Re \mathrm{e}(\sigma)$ and $\Im \mathrm{m}(\sigma)$ as a function of the wavenumber $k$ for the most dangerous mode. The thickness is constant $(h=12.8)$ and the mean density is increased: $\bar{\nu}=0.08(\mathrm{~A}), \bar{\nu}=0.13(\mathrm{~B})$ and $\bar{\nu}=0.16(\mathrm{C})$ (see the stability diagram in figure $\overline{1}$ ). $e=0.6$ and $\phi=0.05$.

length $\lambda_{c}$ strongly varies along the marginal curve for the propagating instability. In figure 6 we have plotted $\lambda_{c} / h$ as a function of $h$. We observe that $\lambda_{c}$ is about 2.5 the thickness $h$ of the flow as long as $h<15$ particle diameters but starts to increase for large thickness. For example, $h=21$ correspond to a wavelength $\lambda_{c}=315$, which is 15 times the thickness of the flow. We shall discuss later this behavior as the signature of a change in the instability mechanism.

\subsection{Eigenfunctions}

The three-dimensional stability analysis of the basic flow gives also the five eigenfunctions $(\hat{\nu}(z), \hat{u}(z), \hat{v}(z), \hat{w}(z), \hat{T}(z))$ for a given wavenumber $k$ and growth rate $\sigma$. In figure 0 , we have plotted the real part of the eigenfunctions corresponding to the most amplified mode for $(h=8, \bar{\nu}=0.23)$. This mode is stationary. We observe that the perturbation of density $\hat{\nu}(z)$ vanishes for $z>h$ whereas the velocity and temperature perturbations 


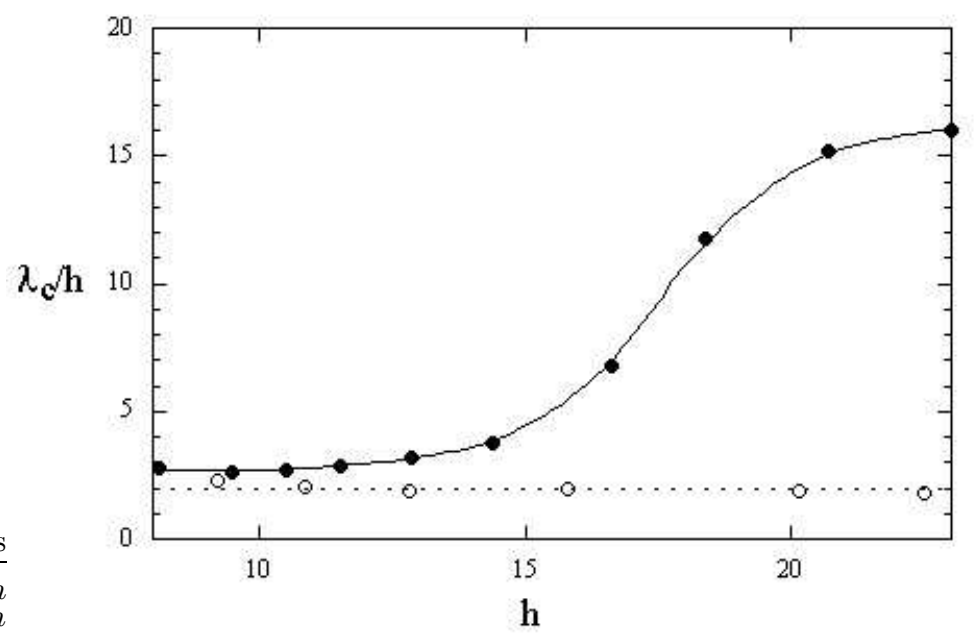

FiguRE $6 . \lambda_{c} / h(\bullet)$ as a function of $h$, where $\lambda_{c}$ is the wavelength selected by the instability along the marginal curve for the propagating instability. The white circles (o) is the same curve obtained when the collisional dissipation $\gamma$ is artificially set to zero. $(e=0.6, \phi=0.05)$.

extend further and decay exponentially (see appendix B). From these eigenfunctions, we can simply recover the perturbed field $\left(\nu_{1}, u_{1}, v_{1}, w_{1}, T_{1}\right)(y, z, t)$. For example, $\nu_{1}(y, z, t)$ is obtained from the eigenfunction $\hat{\nu}(z)$ by the relation $\nu_{1}(y, z, t)=\Re e[\hat{\nu}(z) \exp (\sigma t+$ $i k y)$ ] (see equation 4.2). In the following the perturbed field are plotted for $t=0$. In figure 8, we present the perturbations of the flow corresponding to the most amplified stationary mode presented in figure $7(h=8, \bar{\nu}=0.23)$. The pattern is typical of that observed in the stationary unstable region. Figure 8(a) presents the transverse velocity field $\left(v_{1}(y, z, 0), w_{1}(y, z, 0)\right)$. We can see that the motion in the transverse plane consists in counter-rotating vortices with a pair of vortices per wavelength $\lambda$. This transverse flow is strongly correlated with the perturbation of the longitudinal velocity. Figure 8 (b) shows the contours of constant value for the perturbed longitudinal velocity $u_{1}(y, z, 0)$. The material going towards the plane is flowing faster in the slope direction than the material rising up to the free surface. Therefore, the 3D structure of the perturbed flow consists in longitudinal vortices with transverse variation of the longitudinal velocity.

The corresponding perturbations of density $\nu_{1}(y, z, 0)$ and temperature $T_{1}(y, z, 0)$ are given in figure $8(\mathrm{c})$ and $(\mathrm{d})$. The perturbed density results from two effects: the advection of the basic flow by the transverse perturbed flow and the compressibility. In order to compare more easily the density with the transverse flow, we have plotted in figure 9 the depth averaged density $\mu(y)=\int_{0}^{z_{\max }} \nu_{1}(y, z) \mathrm{d} z$ as a function of the transverse direction $y$ (solid line). The vertical velocity is given on the same plot (dotted line). We observe that the average density is higher where the flow is going downwards and smaller when the flow is going upwards. The perturbation of density can also be interpreted in term of free surface perturbation. If we define the free surface as being the surface where the total density $\left(\nu_{0}(z)+\nu_{1}(y, z, 0)\right)$ is constant and equal to $1 \%$ of the maximum value of $\nu_{0}(z)$, we obtain the bold solid line in figure $8(a)$. One can see that the downward part of the flow corresponds to a through while the upward part of the flow corresponds to a crest.

Most of the features of the stationary modes are recovered for the propagating modes except that the whole pattern is now drifting in the transverse direction, due to the nonzero phase velocity (see figure 10). Qualitative differences exist since the propagating 
Stability analysis of rapid granular chute flows: formation of longitudinal vortices. 15

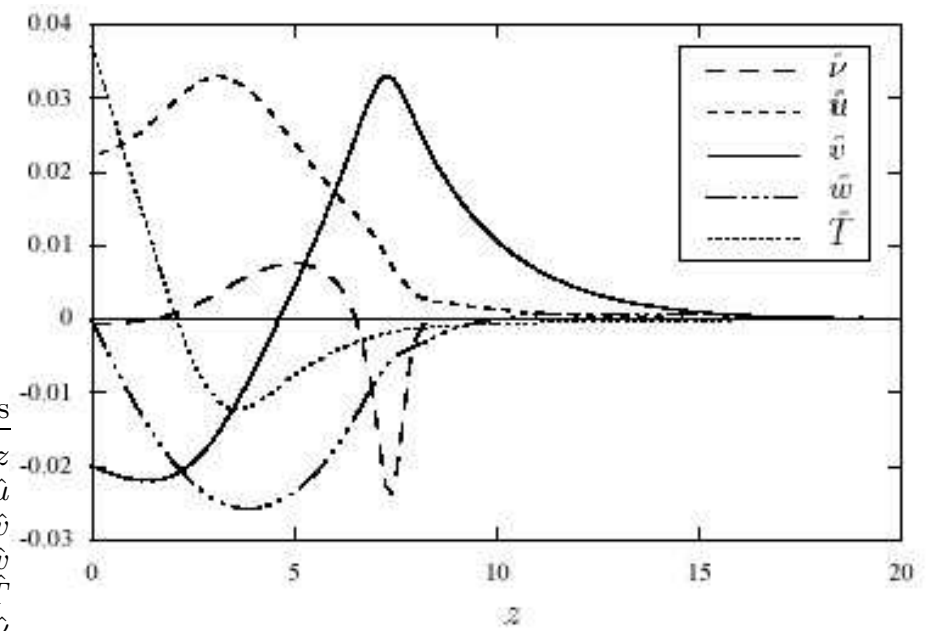

Figure 7. Real part of the eigenfunctions $(\hat{\nu}(z), \hat{u}(z), \hat{v}(z), \hat{w}(z), \hat{T}(z))$ corresponding to the stationary mode $(h=8, \bar{\nu}=0.23, k=0.4)$. $(e=0.6, \phi=0.05)$.

modes lead to a phase shift between the eigenfunctions. The vortices are asymetric as shown in figure 10(a) and the transverse variations of density and longitudinal velocity are no longer in phase but slightly shifted.

\subsection{Influence of the parameters}

The results of the stability analysis presented in the previous section have been performed using a given set of parameters $e=0.6$ and $\phi=0.05$. Changing these parameters modifies the solutions for steady uniform flows and therefore modifies the results of the stability analysis. However, the main results of the previous section are not qualitatively changed in the range of parameters $(e, \phi)$ where inverted density profiles are observed. For instance, we have perform the stability analysis using $e=0.8$ and $\phi=0.12$ and recover the instability and the formation of longitudinal vortices. The domain of propagating instability increases with higher values of $\phi$ and the vortices are more tilted from the vertical for the propagating unstable modes (see figure 11).

However, an important difference with the case $(e=0.6, \phi=0.05)$ is that the curve of marginal stability may overlap the domain of non-inverted density profiles. This means that for some values of $\phi$ and $e$, it is possible to find basic flows with non-inverted density profiles which are unstable. These non-inverted unstable flows are observed in a narrow range of the stability diagram for large thickness $(h>30)$ and small density $(\bar{\nu}<0.1)$. We will see in the next section that inelasticity is responsible of the instability of such non-inverted density profiles.

\section{Discussion}

\subsection{Instability mechanism and role of the inelasticity}

In figure the domain of non-inverted density profiles is shown together with the domain of instability. The correlation between the two regions suggests that inversion of the density profile plays an important role in the instability mechanism. We have checked the role of the gravity by artificially modifying $\mathbf{g}$ in the linearized equations. In a wide range of thickness and mean density, increasing gravity increases the growth rate whereas decreasing gravity stabilizes the flow. In that case, the instability comes from the inversion 
$(\alpha)$

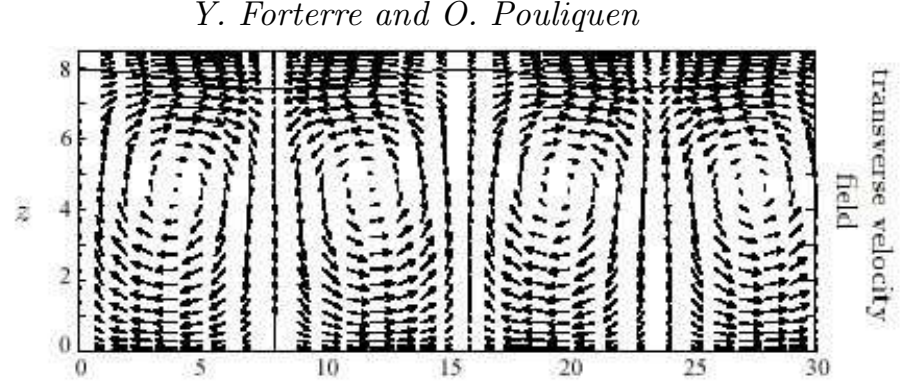

(b)

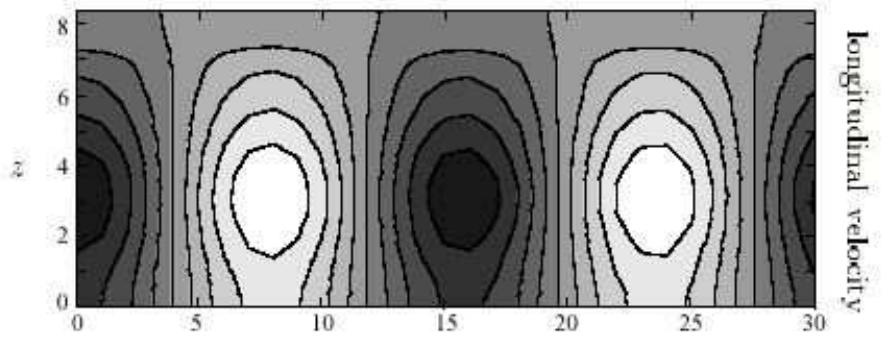

(c)

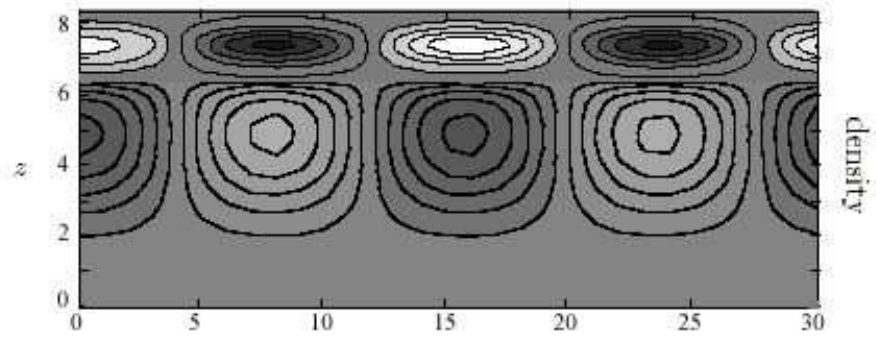

(d)

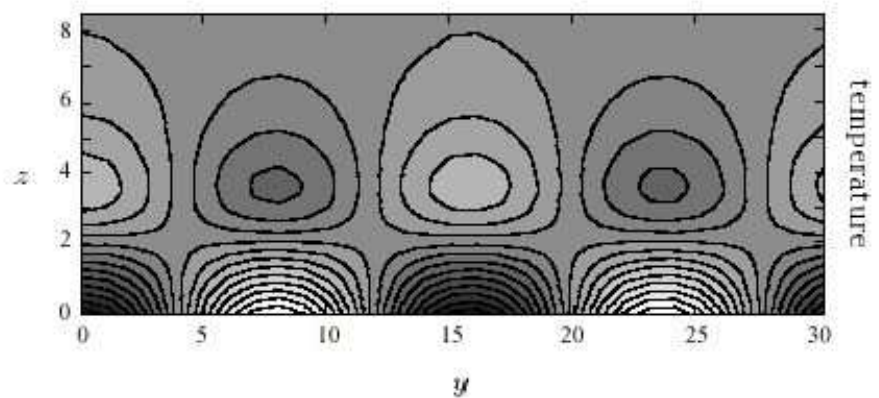

Figure 8. Perturbed fields corresponding to the stationary mode $(h=8, \bar{\nu}=0.23, k=0.4)$; (a) transverse velocity field, the bold solid line gives the free surface deformation; (b), (c), (d) contours of constant values for longitudinal velocity, density and temperature. The grey level is white for the most positive value and black for the most negative value. $(e=0.6, \phi=0.05)$.

of the density profile, which is due to the self-heating at the plane ("Rayleigh-Bénard" type of instability mechanism). In fluid mechanics, the Rayleigh-Bénard instability is controlled at the threshold by a single dimensionless parameter, the Rayleigh number $R_{a}=g \rho \Delta \rho h^{3} / \eta K$. In a granular flow, it is difficult to define such a non-local control parameter since the Boussinesq approximation is far from being satisfied. Indeed, flow quantities (density, temperature) as well as transport coefficients (viscosities, conductivity) strongly vary inside the flow.

As pointed out in the previous section, some flows with non-inverted density profiles are unstable (e.g. with $e=0.8$ and $\phi=0.12$ ). This means that gravity is not the only destabilizing effect. Another well-known source of instability in granular flows is inelasticity. 
Stability analysis of rapid granular chute flows: formation of longitudinal vortices. 17

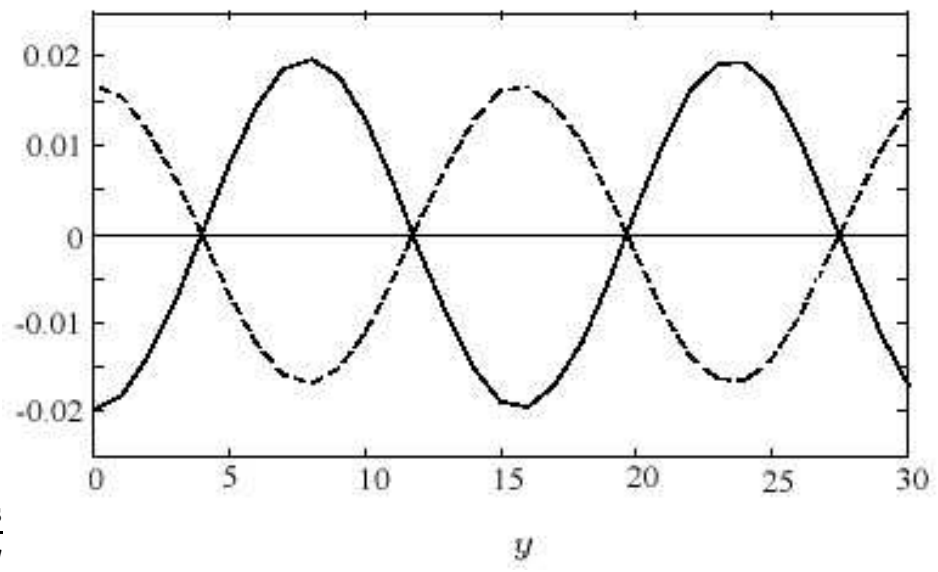

Figure 9. Perturbed depth averaged density $\mu(y)=\int_{0}^{z_{\max }} \nu_{1}(y, z) \mathrm{d} z$ (solid line) as a function of $y$ for the stationary unstable mode $(h=8, \bar{\nu}=0.23, k=0.4)$. The dotted line presents the transverse variations of the perturbed vertical velocity $w_{1}$ (arbitrary scale). The perturbed averaged density is higher where the flow is going downwards $\left(w_{1}<0\right)$ and smaller when the flow is going upwards $\left(w_{1}>0\right)$. $(e=0.6$ and $\phi=0.05)$.

Studies on two-dimensional shear flows have shown that the dissipation due to inelastic collisions can lead to the formation of clusters (Savage 1992 Chi-Hwa Wang et al 1997. Alam \& Nott 1998; Nott et al 1999). In order to better understand the role of dissipation in our problem, we have performed the stability analysis by setting to zero the collisional rate of energy dissipation $\gamma$ in the linearized energy equation. In all the other terms, inelasticity is kept in order to study the same basic flow. Figure 12 presents on the same diagram the curve of marginal stability in the case $\gamma \neq 0$ and $\gamma=0(e=0.6$, $\phi=0.05$ ). We observe that the flow remains unstable in a wide range of parameters with $\gamma=0$. This proves that inelasticity is not necessary to get an instability. For thin and dense flows, inelasticity stabilizes the flow whereas for dilute flows inelasticity slightly lowers the threshold. The interesting point is that non-inverted density profiles are always stable without inelasticity, whatever the choice of parameters $e$ and $\phi$. The dissipation also strongly influences the wavelength selection $\lambda_{c}$ at the threshold. In figure 6 we have plotted $\lambda_{c} / h$ as a function of $h$ both for $\gamma \neq 0$ (black circles) and $\gamma=0$ (white circles). When the dissipation is zero $\gamma=0$, the wavelength at the threshold scales with the thickness of the flow over the whole range of thickness $\left(\lambda_{c} \sim 2 h\right)$. This implies that the increase of $\lambda_{c}$ for large $h$ observed in the real system $(\gamma \neq 0)$ comes from inelasticity.

This analysis suggests that both gravity and inelasticity contribute to the instability depending on the parameters. For thin and dense flows, gravity is the dominant destabilizing effect giving rise to a Rayleigh-Bénard kind of instability, whereas for large and dilute flows inelasticity is the principal ingredient of instability, giving rise to a clusteringlike instability. However, it is not possible to separate the two effects since gravity and inelasticity are associated to the same unstable mode.

\subsection{Comparison with experimental observations}

The present study captures the principal features of the longitudinal vortices instability. First, our analysis has shown that steady uniform flows down inclined planes may be unstable under transverse perturbations. In the range of parameters of the experiment $(h=10-12$ grain diameters, $\bar{\nu}=0.2-0.3)$, the physical origin of this instability is the inversion of the density profile, which is induced by the self-heating at the plane. 
(a)

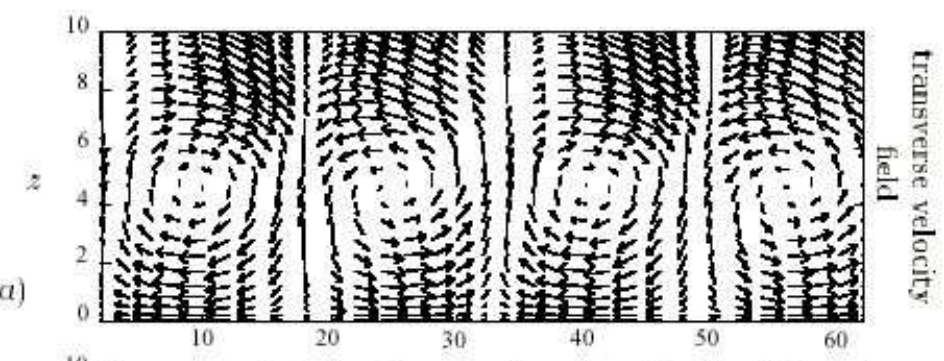

(b)
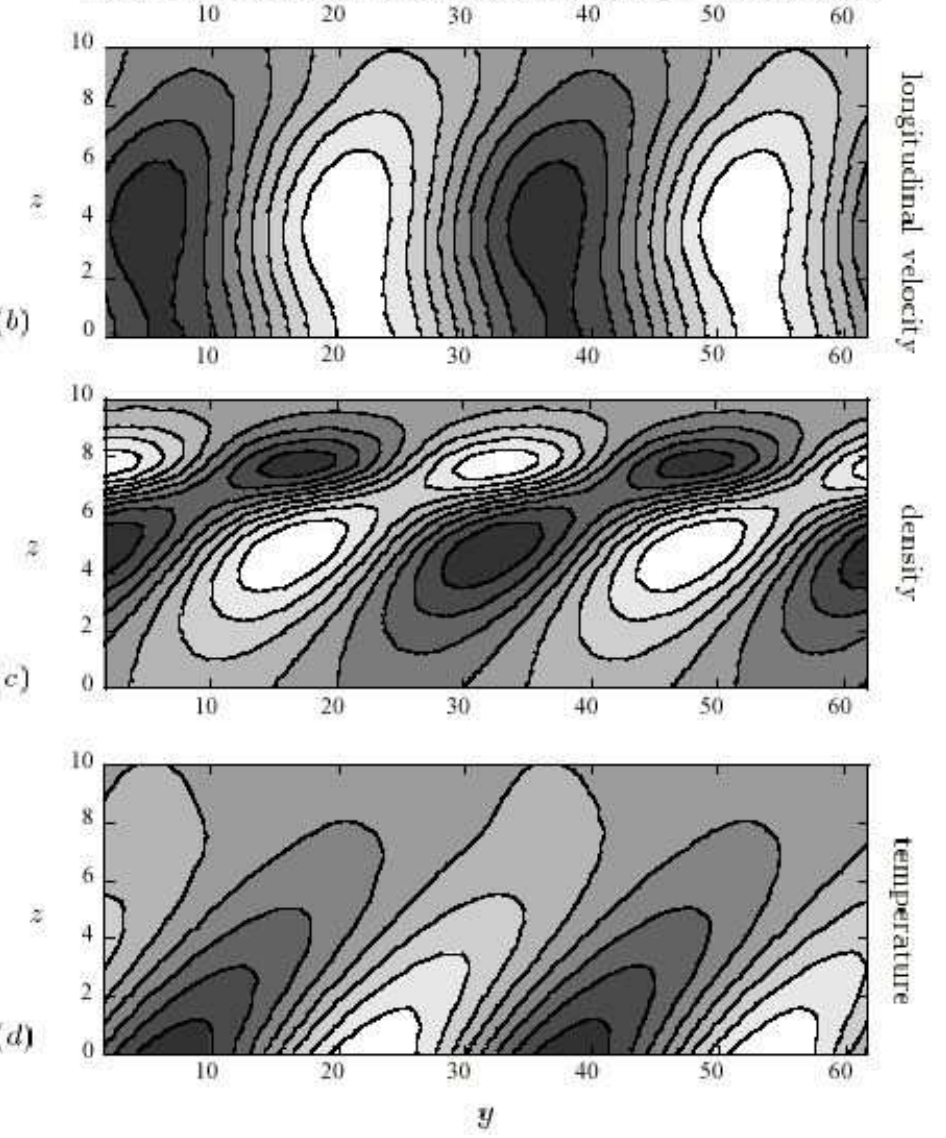

FiguRE 10. Perturbed fields corresponding to the propagating mode $(h=10, \bar{\nu}=0.13, k=0.2)$; (a) transverse velocity field; (b), (c), (d) contours of constant values for longitudinal velocity, density and temperature. $(e=0.6, \phi=0.05)$.

Secondly, the unstable flow consists in longitudinal vortices leading to surface deformation, transverse variations of longitudinal velocity and density in agreement with the experimental observations. As observed in the experiment, the longitudinal velocity is larger in the troughs (i.e. where the flow is going downwards) than in the crests (i.e. where the flow is going upwards). The same variation occurs with the density, troughs are dense and crests are dilute. Finally, the instability selects a wavelength which is always $2-3$ times the average thickness of the flow above the threshold, as observed in the experiment.

It is more difficult to conclude about the relevance of this analysis in order to explain the phase velocity observed in the experiment. We have seen that depending on the control parameters $(h, \bar{\nu})$, the growth rate of the instability may be complex i.e. $\sigma_{i} \neq 0$. 
Stability analysis of rapid granular chute flows: formation of longitudinal vortices. 19

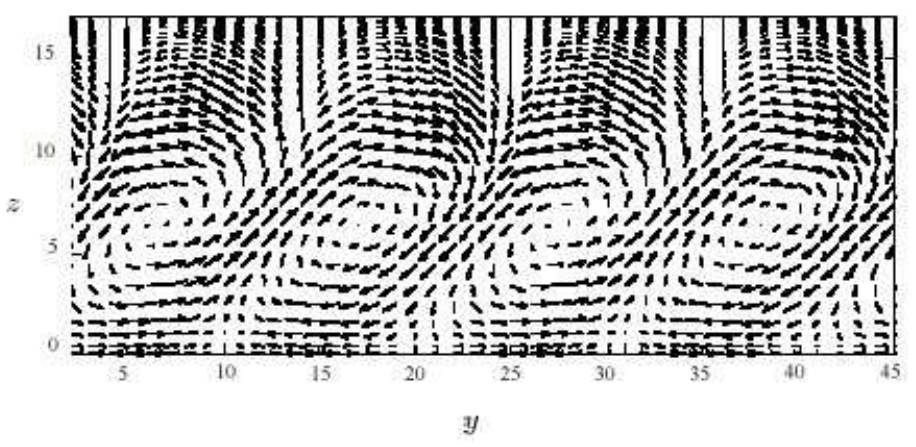

Figure 11. Typical transverse velocity field with $e=0.8$ and $\phi=0.12$ for the propagating instability $(h=17, \bar{\nu}=0.25, k=0.3)$.

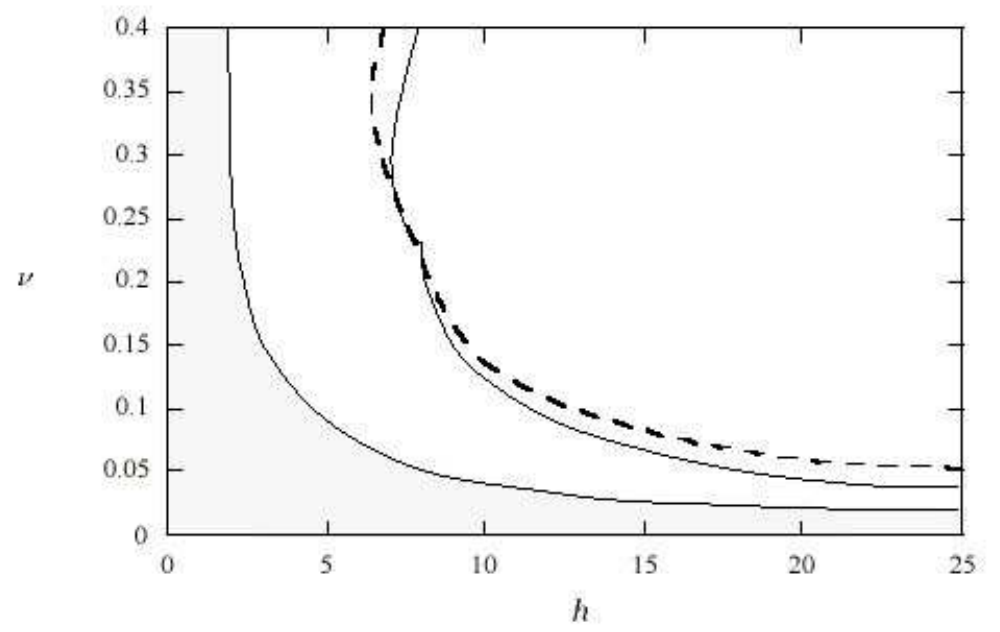

FIgURE 12. Role of inelasticity on the stability diagram. The solid line is the marginal curve with $\gamma \neq 0$ and the dotted line is the marginal curve with $\gamma=0(e=0.6, \phi=0.05)$. The grey zone is the domain of non-inverted density profiles.

This imaginary part of the growth rate is associated to a phase velocity $v_{\phi}=\sigma_{i} / k$, where $k$ is the wavenumber. A typical order of magnitude for $v_{\phi}$ is $(0.1-0.5) \sqrt{g d}$ which is a few percents of the chute velocity. This order of magnitude is compatible with the drift velocity observed in the experiment. However, the theory predicts a transition between propagating and stationary instability, which is not observed in the experiment. Moreover, in the range of parameters of the experiment, the theory predicts a stationary instability. The phase velocity observed experimentally is more likely related to the very weak inclination of the pattern in the experiment. This inclination is not taken into account in this study since we have restricted our analysis to pure transverse perturbations $\left(k_{x}=0, k_{y} \neq 0\right)$. It would be interesting to investigate the stability of steady uniform flows when a small perturbation in the longitudinal direction $\left(k_{x}<<1\right)$ is added to the $k_{y}$ perturbation. This should give rise to a phase velocity in the whole range of parameters.

The agreement between theory and experiment is only qualitative. The most important discrepancy between theory and experiment lies in the range of angles where the instability is predicted. With $e=0.6$ and $\phi=0.05$, the predicted angles are $\theta \sim 20^{\circ}$ 
while in the experiment the minimum angle in order to observe the instability is $\theta=38^{\circ}$ with sand $0.25 \mathrm{~mm}$ diameter. The same order of magnitude is obtained in the experiment with monodisperse glass beads. In the theory, it is not possible to reach such inclination by varying the parameters $(e, \phi)$ except for non-physical values of inelasticity $(e \sim 0)$. Actually, this discrepancy between theory and experiment is not surprising since the standard kinetic theory is known to be limited. The kinetic theory has been first developed for quasi-elastic particles $(e \sim 1)$ and rather dilute flows. Even under these conditions, experimental studies of $2 \mathrm{D}$ collisional granular flows down inclined planes have shown that the theory is not quantitative (Azanza et al 1999). One of the main problem of the kinetic theory is the determination of the boundary conditions at the rough plane. Experiments indicate that, even in the collisional regime, the material is structured near the plane Azanza et al 1999). This local organization is not taken into account in the kinetic theory. Since boundary conditions control the production of granular temperature, it is not surprising that the theory fails in quantitatively describe steady uniform flows. Another difficulty of the kinetic theory lies in the treatment of strongly inelastic particules. For high inelasticity, additional terms should be introduced in the kinetic theory in order to take into account the lack of separation between the microscopic and macroscopic scales inherent to inelasticity (Tan \& Goldhirsch 1999 ; Sela \& Goldhirsch 1998). However, such additional terms complicate considerably the equations and are still the subject of active researches (Goldhirsch 1999).

\section{Conclusion}

In this paper, we have performed a three-dimensional stability analysis of rapid granular flows in the framework of the kinetic theory of granular gases. We have shown that steady uniform flows down inclined planes can be unstable under transverse perturbations. The structure of the unstable flow consists in longitudinal vortices with transverse variations of free surface, chute velocity and density in agreement with the experimental observations (Forterre \& Pouliquen 2001). Actually, the agreement is only qualitative. This is not surprising since the experiments take place in a semi-dilute regime and use rather inelastic particles, which is beyond the domain of applicability of the simple kinetic theory used in the analysis. However, our study shows that the kinetic theory is a relevant framework for the description of rapid granular flows. The kinetic theory has revealed a new instability mechanism specific to granular material: inelastic collisions trigger a self-induced convection yielding longitudinal vortices in chute flows.

In classical fluid mechanics, Rayleigh-Bénard convection is the paradigm for pattern forming instabilities and the study of the transition to turbulence. Therefore, an important question is whether the granular convection observed in our experiment represents the starting point of a similar scenario towards more complex structures. Actually, we have observed in the experiment a new pattern when the plane is strongly inclined $\left(\theta>50^{\circ}\right.$ with sand $0.25 \mathrm{~mm}$ in mean diameter). Instead of longitudinal streaks, a regular square pattern looking as "fish scales" develops on the free surface, as shown in figure 13. Note that under such inclinations, a fast camera is necessary to capture this structure. The appearance of this new pattern raises several issues. A first possibility is that for this range of parameters the square pattern represents the most unstable mode for the primary instability, as for example observed in inclined layer convection (Daniels et al 2000). This could be investigated by generalizing the present stability analysis to longitudinal modes i.e. $k_{x} \neq 0$ and $k_{y} \neq 0$. A second possibility is that the "scales" result from a secondary instability of the longitudinal vortices, as a consequence of nonlinear effects. 
Stability analysis of rapid granular chute flows: formation of longitudinal vortices. 21

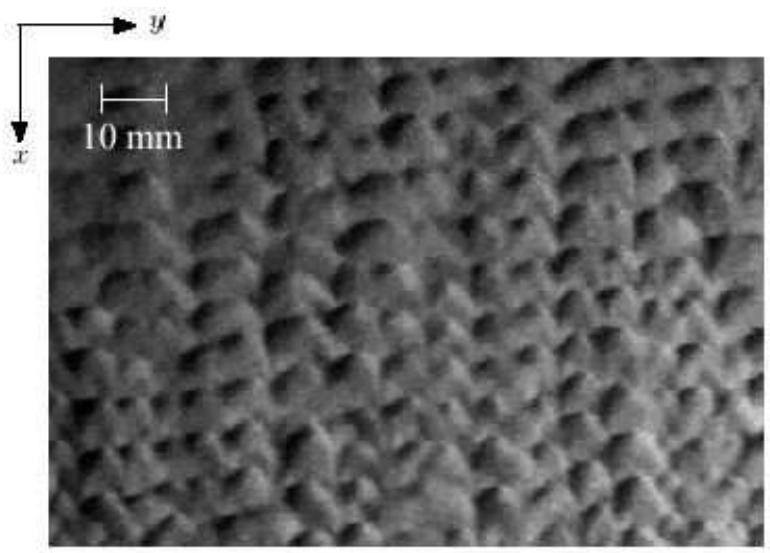

Figure 13. Top view of the free surface of the flow showing the formation of "scales" when the plane is strongly inclined (sand $0.25 \mathrm{~mm}$ mean diameter, $\theta=52^{\circ}, h_{g}=15 \mathrm{~mm}$ ). The picture is taken with a short shutter time of $1 / 10000 s$.

Such an evolution is well-documented for classical fluid flows (Godrèche \& Manneville 1998) but still remains an open issue for granular flows.

This work was supported by the French Ministry of Research and Éducation (ACI blanche \#2018). We thank Stéphane Le Dizès and Jacques Liandrat for fruitful discussions.

\section{Appendix A.}

In this Appendix we detail the main steps of the linear stability analysis presented in Section 4.1 .

\section{A.1. linearization of the governing equations}

The linearisation of the mass equation (2.1), the momentum equation (2.2) and the energy equation (2.3) around the basic flow $\left(\nu_{0}, u_{0}, T_{0}\right)$ gives:

$$
\begin{aligned}
& \frac{\partial \nu_{1}}{\partial t}=-\nu_{0}\left(\frac{\partial v_{1}}{\partial y}+\frac{\partial w_{1}}{\partial z}\right)-\frac{\mathrm{d} u_{0}}{\mathrm{~d} z} w_{1}, \\
& \nu_{0}\left(\frac{\partial u_{1}}{\partial t}+\frac{\mathrm{d} u_{0}}{\mathrm{~d} z} w_{1}\right)=\nu_{1} \sin \theta+\frac{\partial \Sigma_{x y_{1}}}{\partial y}+\frac{\partial \Sigma_{x z 1}}{\partial z}, \\
& \nu_{0} \frac{\partial v_{1}}{\partial t}=\frac{\partial \Sigma_{y y_{1}}}{\partial y}+\frac{\partial \Sigma_{y z_{1}}}{\partial z}, \\
& \nu_{0} \frac{\partial w_{1}}{\partial t}=-\nu_{1} \cos \theta+\frac{\partial \Sigma_{z y_{1}}}{\partial y}+\frac{\partial \Sigma_{z z 1}}{\partial z}, \\
& \frac{3}{2} \nu_{0}\left(\frac{\partial T_{1}}{\partial t}+\frac{\mathrm{d} T_{0}}{\mathrm{~d} z} w_{1}\right)=\Sigma_{x z 1} \frac{\mathrm{d} u_{0}}{\mathrm{~d} z}+\Sigma_{x z 0} \frac{\partial u_{1}}{\partial z}+\Sigma_{y y_{0}} \frac{\partial v_{1}}{\partial y}+\Sigma_{z z 0} \frac{\partial w_{1}}{\partial y} \\
&-\frac{\partial q_{y_{1}}}{\partial y}-\frac{\partial q_{z 1}}{\partial z}-\gamma_{1} .
\end{aligned}
$$


where $\boldsymbol{\Sigma}_{1}$ is the stress tensor disturbance, $\mathbf{q}_{1}$ is the heat flux disturbance and $\gamma_{1}$ is the energy dissipation disturbance given by:

$$
\begin{aligned}
\Sigma_{x y_{1}} & =\eta_{0} \frac{\partial u_{1}}{\partial y} \\
\Sigma_{x z 1} & =\eta_{0} \frac{\partial u_{1}}{\partial z}+\eta_{1} \frac{\mathrm{d} u_{0}}{\mathrm{~d} z} \\
\Sigma_{y y_{1}} & =-P_{1}+\left(\xi_{0}-\frac{2}{3} \eta_{0}\right)\left(\frac{\partial v_{1}}{\partial y}+\frac{\partial w_{1}}{\partial z}\right)+2 \eta_{0} \frac{\partial v_{1}}{\partial y} \\
\Sigma_{y z_{1}} & =\eta_{0}\left(\frac{\partial v_{1}}{\partial z}+\frac{\partial w_{1}}{\partial y}\right) \\
\Sigma_{z z 1} & =-P_{1}+\left(\xi_{0}-\frac{2}{3} \eta_{0}\right)\left(\frac{\partial v_{1}}{\partial y}+\frac{\partial w_{1}}{\partial z}\right)+2 \eta_{0} \frac{\partial w_{1}}{\partial z} \\
q_{y_{1}} & =K_{0} \frac{\partial T_{1}}{\partial y} \\
q_{z_{1}} & =K_{0} \frac{\partial T_{1}}{\partial z}+K_{1} \frac{\mathrm{d} T_{0}}{\mathrm{~d} z} .
\end{aligned}
$$

Here the pressure disturbance $P_{1}$, the viscosity disturbance $\eta_{1}$, the conductivity disturbance $K_{1}$ and the energy dissipated disturbance $\gamma_{1}$ are linearized functions of $\nu_{1}$ and $T_{1}$ which are given in table 2 .

The system of equations (A 1)- A 5 ) must be considered together with the boundary conditions for the disturbances $\left(\nu_{1}(z), u_{1}(z), v_{1}(z), w_{1}(z), T_{1}(z)\right)$. At the plane, the boundary conditions are written as:

$$
\begin{aligned}
w_{1} & =0 \quad \text { at } z=0, \\
\eta_{0} \frac{\partial u_{1}}{\partial z}+\eta_{1} \frac{\mathrm{d} u_{0}}{\mathrm{~d} z} & =\eta_{0}{ }^{*} u_{1}+\eta_{1}{ }^{*} u_{0} \quad \text { at } z=0, \\
\eta_{0}\left(\frac{\partial v_{1}}{\partial z}+\frac{\partial w_{1}}{\partial y}\right) & =\eta_{0}{ }^{*} v_{1} \quad \text { at } z=0, \\
-K_{0} \frac{\partial T_{1}}{\partial z}-K_{1} \frac{\mathrm{d} T_{0}}{\mathrm{~d} z} & =\eta_{1} \frac{\mathrm{d} u_{0}}{\mathrm{~d} z} u_{0}+\eta_{0} u_{0} \frac{\partial u_{1}}{\partial z}+\eta_{0} \frac{\mathrm{d} u_{0}}{\mathrm{~d} z} u_{1}+K_{1}{ }^{*} \quad \text { at } z=0,
\end{aligned}
$$

where $\eta_{1}{ }^{*}$ and $K_{1}{ }^{*}$ are given in table 2. The first condition (A 6) simply expresses that the plane is rigid. The last three conditions (A 7)-(A 9) are the boundary conditions (2.10) and (2.11), which are linearized around the basic flow $\left(\nu_{0}, u_{0}, T_{0}\right)$. At infinity, we assume the disturbances to vanish:

$$
\nu_{1}, u_{1}, v_{1}, w_{1}, T_{1} \rightarrow 0 ; \quad \text { when } z \rightarrow \infty
$$

\section{A.2. Matrices of the eigenvalue problem}

The non-zero elements of the coefficient matrices in equations (4.3) and (4.4) are given below. The definition of the functions of the basic flow $\left(a_{0}, \ldots, r_{0}\right)$ are given in table 2 .

$$
\begin{aligned}
L_{22} & =\eta_{0}, \\
L_{33} & =\eta_{0}, \\
L_{44} & =\xi_{0}+\frac{4}{3} \eta_{0}, \\
L_{55} & =K_{0},
\end{aligned}
$$




$$
\begin{aligned}
P_{1} & =f_{1}{ }^{\prime}\left(\nu_{0}\right) T_{0} \nu_{1}+f_{1}\left(\nu_{0}\right) T_{1}=a_{0} \nu_{1}+b_{0} T_{1} \\
\eta_{1} & =f_{2}{ }^{\prime}\left(\nu_{0}\right) T_{0}{ }^{\frac{1}{2}} \nu_{1}+\frac{1}{2} f_{2}\left(\nu_{0}\right) T_{0}{ }^{-\frac{1}{2}} T_{1}=c_{0} \nu_{1}+d_{0} T_{1} \\
K_{1} & =f_{3}{ }^{\prime}\left(\nu_{0}\right) T_{0}{ }^{\frac{1}{2}} \nu_{1}+\frac{1}{2} f_{3}\left(\nu_{0}\right) T_{0}{ }^{-\frac{1}{2}} T_{1}=e_{0} \nu_{1}+h_{0} T_{1} \\
\gamma_{1} & =f_{5}{ }^{\prime}\left(\nu_{0}\right) T_{0}{ }^{\frac{3}{2}} \nu_{1}+\frac{3}{2} f_{5}\left(\nu_{0}\right) T_{0}{ }^{\frac{1}{2}} T_{1}=l_{0} \nu_{1}+m_{0} T_{1} \\
\eta_{1}{ }^{*} & =\phi f_{6}{ }^{\prime}\left(\nu_{0}\right) T_{0}{ }^{\frac{1}{2}} \nu_{1}+\frac{1}{2} \phi f_{6}\left(\nu_{0}\right) T_{0}{ }^{-\frac{1}{2}} T_{1}=n_{0} \nu_{1}+p_{0} T_{1} \\
\gamma_{1}{ }^{*} & =\left(1-e^{2}\right) f_{7}{ }^{\prime}\left(\nu_{0}\right) T_{0}{ }^{\frac{3}{2}} \nu_{1}+\frac{3}{2}\left(1-e^{2}\right) f_{7}\left(\nu_{0}\right) T_{0}{ }^{\frac{1}{2}} T_{1}=q_{0} \nu_{1}+r_{0} T_{1}
\end{aligned}
$$

TABLE 2. Pressure disturbance $P_{1}$, viscosity disturbance $\eta_{1}$, conductivity disturbance $K_{1}$ and energy dissipated disturbance $\gamma_{1}$

$$
\begin{aligned}
& M_{14}=\nu_{0} \\
& M_{21}=c_{0} \frac{\mathrm{d} u_{0}}{\mathrm{~d} z} \\
& M_{22}=\frac{\mathrm{d} \eta_{0}}{\mathrm{~d} z} \\
& M_{25}=d_{0} \frac{\mathrm{d} u_{0}}{\mathrm{~d} z} \\
& M_{33}=\frac{\mathrm{d} \eta_{0}}{\mathrm{~d} z} \\
& M_{34}=i k\left(\xi_{0}+\frac{1}{3} \eta_{0}\right), \\
& M_{41}=-a_{0} \\
& M_{43}=i k\left(\xi_{0}+\frac{1}{3} \eta_{0}\right), \\
& M_{44}=\frac{\mathrm{d} \xi_{0}}{\mathrm{~d} z}+\frac{4}{3} \frac{\mathrm{d} \eta_{0}}{\mathrm{~d} z}, \\
& M_{45}=-b_{0} \\
& M_{51}=e_{0} \frac{\mathrm{d} T_{0}}{\mathrm{~d} z}, \\
& M_{52}=2 \eta_{0} \frac{\mathrm{d} u_{0}}{\mathrm{~d} z}, \\
& M_{54}=-P_{0}, \\
& M_{55}=\frac{\mathrm{d} K_{0}}{\mathrm{~d} z}+h_{0} \frac{\mathrm{d} T_{0}}{\mathrm{~d} z}, \\
& N_{11}=\sigma \\
& N_{13}=i k \nu_{0} \\
& N_{14}=\frac{\mathrm{d} u_{0}}{\mathrm{~d} z}, \\
& N_{21}=\sin \theta+c_{0} \frac{\mathrm{d}^{2} u_{0}}{\mathrm{~d} z^{2}}+\frac{\mathrm{d} c_{0}}{\mathrm{~d} z} \frac{\mathrm{d} u_{0}}{\mathrm{~d} z},
\end{aligned}
$$




$$
\begin{aligned}
& N_{22}=-\sigma \nu_{0}-k^{2} \eta_{0}, \\
& N_{24}=-\nu_{0} \frac{\mathrm{d} u_{0}}{\mathrm{~d} z}, \\
& N_{25}=d_{0} \frac{\mathrm{d}^{2} u_{0}}{\mathrm{~d} z^{2}}+\frac{\mathrm{d} d_{0}}{\mathrm{~d} z} \frac{\mathrm{d} u_{0}}{\mathrm{~d} z}, \\
& N_{31}=-i k a_{0} \text {, } \\
& N_{33}=-\sigma \nu_{0}-k^{2}\left(\xi_{0}+\frac{4}{3} \eta_{0}\right) \text {, } \\
& N_{34}=i k \frac{\mathrm{d} \eta_{0}}{\mathrm{~d} z}, \\
& N_{35}=-i k b_{0} \text {, } \\
& N_{41}=-\cos \theta-\frac{\mathrm{d} a_{0}}{\mathrm{~d} z}, \\
& N_{43}=i k\left(\frac{\mathrm{d} \xi_{0}}{\mathrm{~d} z}-\frac{2}{3} \frac{\mathrm{d} \eta_{0}}{\mathrm{~d} z}\right), \\
& N_{44}=-\sigma \nu_{0}-k^{2} \eta_{0} \text {, } \\
& N_{45}=-\frac{\mathrm{d} b_{0}}{\mathrm{~d} z} \text {, } \\
& N_{51}=c_{0}\left(\frac{\mathrm{d} u_{0}}{\mathrm{~d} z}\right)^{2}+e_{0} \frac{\mathrm{d}^{2} T_{0}}{\mathrm{~d} z^{2}}+\frac{\mathrm{d} e_{0}}{\mathrm{~d} z} \frac{\mathrm{d} T_{0}}{\mathrm{~d} z}-l_{0}, \\
& N_{53}=-i k P_{0} \text {, } \\
& N_{54}=-\frac{3}{2} \nu_{0} \frac{\mathrm{d} T_{0}}{\mathrm{~d} z}, \\
& N_{55}=-\frac{3}{2} \nu_{0} \sigma-k^{2} K_{0}+d_{0}\left(\frac{\mathrm{d} u_{0}}{\mathrm{~d} z}\right)^{2}+h_{0} \frac{\mathrm{d}^{2} T_{0}}{\mathrm{~d} z^{2}}+\frac{\mathrm{d} h_{0}}{\mathrm{~d} z} \frac{\mathrm{d} T_{0}}{\mathrm{~d} z}-m_{0}, \\
& Q_{22}=\eta_{0}, \\
& Q_{33}=\eta_{0} \text {, } \\
& Q_{52}=-\eta_{0} u_{0} \text {, } \\
& Q_{55}=-K_{0} \text {, } \\
& R_{14}=1 \text {, } \\
& R_{21}=c_{0} \frac{\mathrm{d} u_{0}}{\mathrm{~d} z}-u_{0} n_{0}, \\
& R_{22}=-\eta_{0}{ }^{*} \text {, } \\
& R_{33}=-\eta_{0}{ }^{*} \text {, } \\
& R_{34}=i k \eta_{0}, \\
& R_{51}=-e_{0} \frac{\mathrm{d} T_{0}}{\mathrm{~d} z}-c_{0} u_{0} \frac{\mathrm{d} u_{0}}{\mathrm{~d} z}+q_{0}, \\
& R_{52}=-\eta_{0} \frac{\mathrm{d} u_{0}}{\mathrm{~d} z}, \\
& R_{55}=-h_{0} \frac{\mathrm{d} T_{0}}{\mathrm{~d} z}-d_{0} u_{0} \frac{\mathrm{d} u_{0}}{\mathrm{~d} z}+r_{0} .
\end{aligned}
$$


Stability analysis of rapid granular chute flows: formation of longitudinal vortices. 25

\section{Appendix B.}

Here we give the asymptotic behaviour of the disturbances $(\hat{\nu}, \hat{u}, \hat{v}, \hat{w}, \hat{T})$ when $z$ is much larger than the characteristic thickness of the basic flow. The linearized equations (4.3) which govern the disturbances can be written as

$$
\begin{aligned}
\sigma \hat{\nu}= & -\nu_{0}\left(i k \hat{v}+\frac{\mathrm{d} \hat{w}}{\mathrm{~d} z}\right)-\frac{\mathrm{d} u_{0}}{\mathrm{~d} z} \hat{w}, \\
\nu_{0}\left(\sigma \hat{u}+\frac{\mathrm{d} u_{0}}{\mathrm{~d} z} \hat{w}\right)= & \hat{\nu} \sin \theta-k^{2} \eta_{0} \hat{u}+\frac{\mathrm{d}}{\mathrm{d} z}\left(\eta_{0} \frac{\mathrm{d} \hat{u}}{\mathrm{~d} z}+\hat{\eta} \frac{\mathrm{d} u_{0}}{\mathrm{~d} z}\right), \\
\nu_{0} \sigma \hat{v}= & -i k \hat{P}+\left(\xi_{0}-\frac{2}{3} \eta_{0}\right)\left(-k^{2} \hat{v}+i k \frac{\mathrm{d} \hat{w}}{\mathrm{~d} z}\right)-2 \eta_{0} k^{2} \hat{v} \\
& +\frac{\mathrm{d}}{\mathrm{d} z}\left(\eta_{0} \frac{\mathrm{d} \hat{v}}{\mathrm{~d} z}+i k \eta_{0} \hat{w}\right), \\
\nu_{0} \sigma \hat{w}= & -\hat{\nu} \cos \theta-\frac{\mathrm{d} \hat{P}}{\mathrm{~d} z}+\eta_{0}\left(i k \frac{\mathrm{d} \hat{v}}{\mathrm{~d} z}-k^{2} \hat{w}\right)+\frac{\mathrm{d}}{\mathrm{d} z}\left[\left(\xi_{0}-\frac{2}{3} \eta_{0}\right)\left(i k \hat{v}+\frac{\mathrm{d} \hat{w}}{\mathrm{~d} z}\right)\right] \\
& +2 \frac{\mathrm{d}}{\mathrm{d} z}\left(\eta_{0} \frac{\mathrm{d} \hat{w}}{\mathrm{~d} z}\right), \\
\frac{3}{2} \nu_{0}\left(\sigma \hat{T}+\frac{\mathrm{d} T_{0}}{\mathrm{~d} z} \hat{w}\right)= & \left(\eta_{0} \frac{\mathrm{d} \hat{u}}{\mathrm{~d} z}+\hat{\eta} \frac{\mathrm{d} u_{0}}{\mathrm{~d} z}\right) \frac{\mathrm{d} u_{0}}{\mathrm{~d} z}+\eta_{0} \frac{\mathrm{d} u_{0}}{\mathrm{~d} z} \frac{\mathrm{d} \hat{u}}{\mathrm{~d} z}-P_{0}\left(i k \hat{v}+\frac{\mathrm{d} \hat{w}}{\mathrm{~d} z}\right)-k^{2} K_{0} \hat{T} \\
& +\frac{\mathrm{d}}{\mathrm{d} z}\left(K_{0} \frac{\mathrm{d} \hat{T}}{\mathrm{~d} z}+\hat{K} \frac{\mathrm{d} T_{0}}{\mathrm{~d} z}\right)-\hat{\gamma} .
\end{aligned}
$$

In order to obtain the asymptotic expression of these equations, one has to know the asymptotic behaviour of the basic flow. Equation (3.5) shows that the zero-order density $\nu_{0}$ decays exponentially to zero as

$$
\nu_{0} \rightarrow \exp \left(-\frac{\cos \theta}{T_{0}} z\right)
$$

when $z$ is large. Using equations (3.5)-(3.7), the asymptotic behaviour of the basic flow is given by

$$
\begin{aligned}
\frac{\mathrm{d} u_{0}}{\mathrm{~d} z} & \sim \nu_{0} ; \frac{\mathrm{d}^{2} u_{0}}{\mathrm{~d} z^{2}} \sim \nu_{0}, \\
\frac{\mathrm{d} T_{0}}{\mathrm{~d} z} & \rightarrow 0 ; \frac{\mathrm{d}^{2} T_{0}}{\mathrm{~d} z^{2}} \rightarrow 0, \\
\eta_{0} & \rightarrow C ; \frac{\mathrm{d} \eta_{0}}{\mathrm{~d} z} \rightarrow 0, \\
K_{0} & \rightarrow D ; \frac{\mathrm{d} K_{0}}{\mathrm{~d} z} \rightarrow 0, \\
P_{0} & \sim \nu_{0}, \\
\xi_{0} & \sim \nu_{0}^{2} ; \frac{\mathrm{d} \xi_{0}}{\mathrm{~d} z} \sim \nu_{0}^{2},
\end{aligned}
$$

Considering (B6)-( $\mathrm{B} 11)$ into the linearized equations $(\mathrm{B} 1 \mathrm{1})-(\mathrm{B} 5)$ gives an asymptotic behaviours for $z$ which is much larger than the characteristic thickness of the basic flow. From equation ( $\mathrm{B} 1)$, one shows that the perturbed density decays on the same length scale as the basic flow:

$$
\hat{\nu} \sim \nu_{0}
$$


Therefore, the asymptotic limit of equations $(\mathrm{B} 1)-(\mathrm{B} 5)$ is given by

$$
\begin{aligned}
\frac{\mathrm{d}^{2} \hat{u}}{\mathrm{~d} z^{2}}-k^{2} \hat{u} & =0 \\
\frac{\mathrm{d}^{2} \hat{v}}{\mathrm{~d} z^{2}}-\frac{4}{3} k^{2} \hat{v}+\frac{1}{3} i k \frac{\mathrm{d} \hat{w}}{\mathrm{~d} z} & =0 \\
\frac{1}{3} i k \frac{\mathrm{d} \hat{v}}{\mathrm{~d} z}+\frac{4}{3} \frac{\mathrm{d}^{2} \hat{w}}{\mathrm{~d} z^{2}}-k^{2} \hat{w} & =0 \\
\frac{\mathrm{d} \hat{T}}{\mathrm{~d} z}-k^{2} \hat{T} & =0 .
\end{aligned}
$$

These equations are easily solved and give

$$
\left(\begin{array}{c}
\hat{u} \\
\hat{v} \\
\hat{w} \\
\hat{T}
\end{array}\right) \sim \exp (-k z) .
$$

when $z$ is much larger than the characteristic thickness of the basic flow.

\section{REFERENCES}

Alam, M. \& Nott, P. R. 1998 Stability of plane Couette flow of a granular material. J. Fluid Mech. 377, 99-136.

Anderson, K. G. \& JACKson, R. 1992 A comparison of the solutions of some proposed equations of motion of granular materials for fully developed flow down inclined planes. $J$. Fluid Mech. 241, 145-168.

AzAnZA, E. 1998 Écoulements granulaires bidimensionnels sur un plan incliné. PhD Dissertation, École Nationale des Ponts et Chaussés, France.

Azanza, E., Chevoir, F. \& Moucheront, P. 1999 Experimental study of collisional granular flows down an inclined plane. J. Fluid Mech. 400, 199-227.

Ahn, H., Brennen, C. E. \& Sabersky, R. H. 1992 Analysis of the fully developed chute flow of granular materials. Trans. ASME J. App. Mech. 59, 109-119.

Brey, J. J., Dufty, J. W., Kim, C. S. \& Santos, A. 1998 Hydrodynamics for granular flow at low density. Phys. Rev. E 58, 4638-4653.

Campbell, C. S. 1990 Rapid granular flows. Annu. Rev. Fluid Mech. 22, 57-92.

Campbell, C. S. \& Brennen, C. E. 1985 Chute flows of granular material: some computer simulation. Trans. ASME J. Appl. Mech. 52, 172-178.

Chapman, S. \& Cowling, T. G. 1970 The Mathematical Theory of Non-Uniform Gases, 3rd edn. Cambridge University Press.

Chi-Hwa Wang, Jackson, R. \& Sundaresan, S. 1997 Instabilities of fully developed rapid flow of a granular material in a channel. J. Fluid Mech. 342, 179-197.

Daniels, K. E., Brendan, B. P. \& Bodenschatz, E. 2000 Pattern formation in inclined layer convection. Phys. Rev. Lett. 84, 5320-5323.

Drazin, P. G. \& Reid, W. H. 1981 Hydrodynamic stability. Cambridge University Press.

Falcon, E., FAuve, S. \& Laroche, C. 1999 Cluster formation, pressure and density measurements in a granular medium fluidized by vibration. Eur. Phys. J. B. 9, 183-186.

Forterre, Y. \& Pouliquen, O. 2001 Longitudinal vortices in granular flows. Phys. Rev. Lett. 86, $5886-5889$.

Godrèche, C. \& Manneville, P. 1998 Hydrodynamics and Nonlinear Instabilities. Cambridge University Press.

Goldhirsch, I. \& Zanetti, G. 1993 Clustering instability in dissipative gases. Phys. Rev. Lett. 70, 1619-1622.

Goldhirsch, I. 1999 Scales and kinetics of granular flows. Chaos 9, 659-672.

Gottlieb, D., Hussaini, M. Y. \& Orszag, S. A. 1984 Theory and application of spectral 
Stability analysis of rapid granular chute flows: formation of longitudinal vortices. 27 methods. In Spectral methods for partial differential equations (ed. R. G. Voight, D. Gottlieb \& M. Y. Hussaini). Soc. Indus. \& Appl. Math.

Haff, P. K. 1983 Grain flow as a fluid-mechanical phenomenon. J. Fluid Mech. 134, 401-430.

Hui, K., Haff, P. K., Ungar, J. E. \& JaCkson, R. 1984 Boundary conditions for high-shear grains flows. J. Fluid Mech. 145, 223-233.

Jenkins, J. T. \& Savage, S. B. 1983 A theory for the rapid flow of identical, smooth, nearly elastic, spherical particles. J. Fluid Mech. 130, 187-202.

Jenkins, J. T. \& Richman, M. W. 1986 Boundary conditions for plane flows of smooth, nearly elastic, circular disks. J. Fluid Mech. 171, 53-69.

Johnson, P. C., Nott, P. \& Jackson, R. 1990 Frictional-collisional equations of motion for particulate flows and their application to chutes. J. Fluid Mech. 210, 501-535.

Kachanov, Y. S. 1994 Physical mechanisms of laminar-boundary-layer transition. Annu. Rev. Fluid Mech. 26, 411-482.

Lun, C. K. K., Savage, S. B., Jeffrey, D. J. \& Chepurniy, N. 1984 Kinetic theories for granular flow: inelastic particles in Couette flow and slightly inelastic particles in a general flowfield. J. Fluid Mech. 140, 223-256.

MALiK, M. R. 1990 Numerical methods for hypersonic boundary layer stability. J. Comput. Phys. 86, 376-413.

Mayer, E. W. \& Powel, K. G. 1992 Viscous and invicid instabilities of a trailing line vortex. J. Fluid Mech. 245, 191-114.

McNamara, S. \& Young, W. R. 1992 Inelastic collapse and clumping in a one-dimensional granular medium Phys. Fluid A. 4, 496-504.

Nott, P. R., Alam, M., Agrawal, K., Jackson, R. \& Sundaresan, S. 1999 The effect of boundaries on the plane Couette flow of granular materials: a bifurcation analysis. $J$. Fluid Mech. 397, 203-229.

Ogawa, S., Umemura, A. \& Oshima, N. 1980 On the equations of fully fluidized granular materials. Z. angew. Math. Phys. 31, 483-493.

Rouyer, F. \& Menon, N. 2000 Velocity fluctuations in a homogeneous 2D granular gas in steady-state. Phys. Rev. Lett. 85, 3676-3679.

Saric, W. S. 1994 Görtler vortices. Annu. Rev. Fluid Mech. 26, 379-409.

SAvage, S. B. 1992 Instability of unbounded uniform granular shear flow. J. Fluid Mech. 241, 109-123.

Sela, N. \& Goldhirsch, I. 1998 Hydrodynamic equations for rapid flows of smooth inelastic spheres, to Burnett order. J. Fluid Mech. 361, 41-74.

Tan, M-L. \& Goldhirsch, I. 1999 Rapid granular flows as mesoscopic systems. Phys. Rev. Lett. 81, 3022-3025.

Warr, S., Huntley, J. M., \& Jacques, G. T. H. 1995 Fluidization of a two-dimensional granular system: experimental study and scaling behavior. Phys. Rev. E 52, 5583-5595. 